\title{
KOSZUL BICOMPLEXES AND GENERALIZED KOSZUL COM- PLEXES IN PROJECTIVE DIMENSION ONE
}

BOGDAN ICHIM, Universität Osnabrück, FB Mathematik/Informatik, 49069 Osna-

brück, Germany, bogdan.ichim@mathematik.uni-osnabrueck.de

Institute of Mathematics, C.P. 1-764, 70700 Bucharest, Romania,

bogdan.ichim@imar.ro

UDO VETTER, Universität Oldenburg, Institut für Mathematik, 26111 Oldenburg, Germany, vetter@mathematik.uni-oldenburg.de

\begin{abstract}
We describe Koszul type complexes associated with a linear map from any module to a free module, and vice versa with a linear map from a free module to an arbitrary module, generalizing the classical Koszul complexes. Given a short complex of finite free modules, we assemble these complexes to what we call Koszul bicomplexes. They are used in order to investigate the homology of the Koszul complexes in projective dimension one. As in the case of the classical Koszul complexes this homology turns out to be grade sensitive. In a special setup we obtain necessary conditions for a map of free modules to be lengthened to a short complex of free modules.
\end{abstract}

\section{Introduction}

The paper deals with the following problem: let $\mathcal{F} \stackrel{\chi}{\rightarrow} \mathcal{G} \stackrel{\lambda}{\rightarrow} \mathcal{H}$ be a complex of finite free modules over a noetherian ring $R$; in which way does grade $I_{\lambda}$ depend on grade $I_{\chi}$ and on the ranks of $\mathcal{F}, \mathcal{G}, \mathcal{H}$ ? (By $I_{\lambda}$ we denote the ideal of maximal minors of $\lambda$.) If, for example, $\operatorname{rank} \mathcal{F}=1, \operatorname{rank} \mathcal{G}=n$, and $\chi$ is given by a regular sequence $x_{1}, \ldots, x_{n}$ in $R$, i.e. $\chi(1)=\left(x_{1}, \ldots, x_{n}\right)$, then it was proved in [BV4] that grade $I_{\lambda}=n$ is possible if and only if $\operatorname{rank} \mathcal{H}=1$ and $n$ is even. 
Being far away from a satisfactory solution to the the general problem, we shall report here on some approach.

It turns out that there is a close connection to the behavior of the homology of the generalized Koszul complex associated with the induced map $\bar{\lambda}: \operatorname{Cok} \chi \rightarrow \mathcal{H}$. Here we must clear up what we mean by a generalized Koszul complex since even the terminology concerning the classical Koszul complex is not standardized. We refer to the accesses given in [BO2], $\S 10$ or in $[\mathrm{BH}], 1.6$; in $[\mathrm{E}], \mathrm{Ch} .17$ there is a comprehensive presentation of the 'dual' approach.

The Eagon-Northcott family of complexes $\mathcal{C}^{t}(\psi)$ (see [E], A2.6 or [BV2], 2.C), associated with a linear map $\psi: G \rightarrow F$ of finite free modules $G$ and $F$ such that $\operatorname{rank} G \geq \operatorname{rank} F$, generalizes the Koszul complex in a special case. The homology of $\mathcal{C}^{t}(\psi)$ is well-understood. As in the classical case it is grade sensitive with respect to the ideal $I_{\psi}$.

More generally we consider linear maps $\psi: G \rightarrow F$, where only $F$ has to be finite and free (weaker assumptions on $F$ are possible). We construct a family of complexes $\mathcal{C}_{\psi}(t)$ associated with $\psi$ which generalizes both the Eagon-Northcott family of complexes, and the classical Koszul complex. There is a similar 'dual' construction of a family of complexes $\mathcal{D}_{\varphi}(t)$ for a map $\varphi: H \rightarrow G$ from a finite free module $H$ to an arbitrary module $G$. The complexes just mentioned are the generalized Koszul complexes.

If $H \stackrel{\varphi}{\rightarrow} G \stackrel{\psi}{\rightarrow} F$ is a complex, we can compose $\mathcal{C}_{\psi}(t)$ and $\mathcal{D}_{\varphi}(t)$ to the Koszul bicomplex $\mathcal{K}_{., .}(t)$. Setting $H=\mathcal{H}^{*}, G=\mathcal{G}^{*}, F=\mathcal{F}^{*}$ and $\varphi=\lambda^{*}, \psi=\chi^{*}$, we use it to investigate the homology of $\mathcal{C}_{\bar{\lambda}}(t)$. Assume that rank $\mathcal{F} \leq \operatorname{rank} \mathcal{G}$. Then the most satisfactory result (see Theorem 6.10) is obtained if grade $I_{\chi}$ has the greatest possible value $\operatorname{rank} \mathcal{G}-\operatorname{rank} \mathcal{F}+1$. It covers a result of Migliore, Nagel and Peterson (see Proposition 5.1 in [MPN]) who proved it partially for Gorenstein rings $R$, using local cohomology. It also generalizes Theorem 5 in [BV4]. In the case under consideration a full answer to our initial problem is contained in Theorem 6.5. a generalization of Corollary 3 in [BV1].

What can be deduced if grade $I_{\chi}$ has not the greatest possible value (but is not too small)? In this case Theorem 6.1 provides some answer to the question in the beginning. As a consequence we derive a purely numerical criterion for the non-vanishing of a product of matrices (Corollary 6.4).

The paper is based on results of Bruns and Vetter (see [BV3] and [BV4]). They study the homology of the Koszul complex associated with a linear form on a module of projective dimension one, using a Koszul bicomplex construction obtained from a Koszul complex and certain Eagon-Northcott complexes. The idea to build and link Koszul bicomplexes appears also in the paper [HM] of Herzog and Martsinkovsky (see in particular the gluing construction for the residue field of a complete intersection).

The article for the most part consists of considerations and results contained in the thesis [I] of the first author. 


\section{Preliminaries}

In the paper $R$ always denotes a commutative ring. We adopt the following convention for the graded dual of a graded module: if $M=\oplus_{i \geq 0} M_{i}$ is a graded $R$-module, we shall improperly write $M^{*}$ for the graded dual of $M$, that is

$$
M^{*}=M_{g r}^{*}=\oplus_{i \geq 0}\left(M_{i}\right)^{*}
$$

We use it mainly in the case in which $M$ is the symmetric algebra $S(N)$ or the exterior algebra $\bigwedge N$ of an $R$-module $N$. Then

$$
S(N)^{*}=\oplus_{i \geq 0} S_{i}(N)^{*}, \quad(\bigwedge N)^{*}=\oplus_{i \geq 0}\left(\bigwedge^{i} N\right)^{*} .
$$

Correspondingly $M^{* *}$ means the graded bidual $\left(M_{g r}^{*}\right)_{g r}^{*}$ of $M$.

Let $G$ be an $R$-module. The natural graded algebra homomorphism

$$
\theta: \bigwedge G^{*} \rightarrow(\bigwedge G)^{*}
$$

is given by

$$
\theta\left(y_{1}^{*} \wedge \ldots \wedge y_{p}^{*}\right)\left(y_{1} \wedge \ldots \wedge y_{p}\right)=\operatorname{det}\left(y_{j}^{*}\left(y_{i}\right)\right)
$$

for all $y_{1}, \ldots, y_{p} \in G$ and $y_{1}^{*}, \ldots, y_{p}^{*} \in G^{*}$. If $G$ is finitely generated and projective, then $\theta$ is an isomorphism.

Beside the natural left $\bigwedge G$-module structure, the exterior algebra $\bigwedge G$ carries a natural structure as a right $\bigwedge G^{*}$-module: we set

$$
y_{1} \wedge \ldots \wedge y_{n} \leftarrow y_{1}^{*} \wedge \ldots \wedge y_{p}^{*}=\sum_{\sigma} \varepsilon(\sigma) \operatorname{det}_{1 \leq i, j \leq p}\left(y_{j}^{*}\left(y_{\sigma(i)}\right)\right) y_{\sigma(p+1)} \wedge \ldots \wedge y_{\sigma(n)}
$$

for $y_{1}, \ldots, y_{n} \in G$ and $y_{1}^{*}, \ldots, y_{p}^{*} \in G^{*}$, where $\sigma$ runs through the set of permutations of $n$ elements which are increasing on the intervals $[1, p]$ and $[p+1, n]$. In fact, the operation $\leftarrow$ makes $\bigwedge G$ a right $\bigwedge G^{*}$-module (see [BO1], Ch. III, $\S 11.9$ for details).

An easy calculation shows that an element $z^{*} \in G^{*}$ acts like an antiderivation on $\bigwedge G$ in the sense that

$$
(x \wedge y)<z^{*}=\left(x<z^{*}\right) \wedge y+(-1)^{\operatorname{deg} x} x \wedge\left(y<z^{*}\right)
$$

for elements $x, y \in \bigwedge G, x$ homogeneous. By induction we obtain immediately the following associativity formula:

PROPOSITION 2.1. Let $x_{i} \in G$ for $i=1, \ldots, l$, and let $z_{j}^{*} \in G^{*}$ for $j=1, \ldots, p$. Assume that $z_{j}^{*}\left(x_{i}\right)=0$ for all $i, j$. Then

$$
x_{1} \ldots x_{l} \wedge\left(y_{1} \ldots y_{n} \leftarrow z_{1}^{*} \ldots z_{p}^{*}\right)=(-1)^{l p}\left(x_{1} \ldots x_{l} \wedge y_{1} \ldots y_{n}\right) \leftarrow z_{1}^{*} \ldots z_{p}^{*}
$$

for all $y \in \bigwedge G$. 
Furthermore we shall make use of

PROPOSITION 2.2. Let $y^{*} \in \bigwedge G^{*}$ be homogeneous of degree $p$. Let $y^{*} \wedge$ denote the left multiplication by $y^{*}$ on $\Lambda G^{*}$, and $<y^{*}$ the right multiplication by $y^{*}$ on $\Lambda G$. Then the diagram

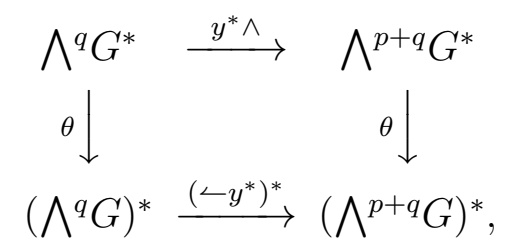

is commutative.

Proof. Let $x \in \bigwedge^{p+q} G$ and $z^{*} \in \bigwedge^{q} G^{*}$. Then

$\theta\left(y^{*} \wedge z^{*}\right)(x)=x\left\llcorner\left(y^{*} \wedge z^{*}\right)=\left(x\left\llcorner y^{*}\right)\left\llcorner z^{*}=\theta\left(z^{*}\right)\left(x\left\llcorner y^{*}\right)=\left(\left\llcorner y^{*}\right)^{*}\left(\theta\left(z^{*}\right)\right)(x)\right.\right.\right.\right.\right.$.

The last paragraph of this preparing section is devoted to the divided power algebra $D(H)$ of a free $R$-module $H$ of finite rank. For the definition we refer to [E], A2.4. Here we only explain the natural structure of $D(H)$ as a graded $S\left(H^{*}\right)$-module which we shall use in the next section.

The natural structure of $S(H)^{*}$ as a graded $S(H)$-module is defined by

$$
\left(a y^{*}\right)(x)=y^{*}(a x)
$$

for all $a, x \in S(H)$ and all $y^{*} \in S(H)^{*}$. Replace $H$ by $H^{*}$, use the canonical equality $H=H^{* *}$, and set

$$
\left(a^{*} c\right)\left(b^{*}\right)=\alpha(c)\left(a^{*} b^{*}\right)
$$

for all $a^{*}, b^{*} \in S\left(H^{*}\right)$ and $c \in D(H)$ where the graded algebra isomorphism $\alpha$ : $D(H) \rightarrow S\left(H^{*}\right)^{*}$ is given by the formula

$$
\alpha\left(h^{(k)}\right)\left(\prod_{i}\left(h_{i}^{*}\right)^{k_{i}}\right)=\left\{\begin{array}{ccc}
0 & \text { if } & \sum k_{i} \neq k \\
\prod_{i}\left(h_{i}^{*}(h)\right)^{k_{i}} & \text { if } & \sum k_{i}=k,
\end{array}\right.
$$

$h \in H, h_{i}^{*} \in H^{*}$ (see [BE2], $\left.1 \mathrm{~A}^{\prime}\right)$ ). The interaction between the $S\left(H^{*}\right)$-module structure of $D(H)$ and the algebra structure of $D(H)$ is described by the following result which we quote without its easy proof.

PROPOSITION 2.3. If $h^{*} \in H^{*}$, then $h^{*}$ acts like a derivation on $D(H)$ in the sense that

(a) $h^{*} h^{(k)}=h^{*}(h) h^{(k-1)}$ for all $h \in H, k \geq 1$,

(b) $h^{*}(c d)=\left(h^{*} c\right) d+c\left(h^{*} d\right)$ for all $c, d \in D(H)$. 


\section{Generalized Koszul Complexes}

Let $H \stackrel{\varphi}{\rightarrow} G$ and $G \stackrel{\psi}{\rightarrow} F$ be homomorphisms of $R$-modules. Most of the results of this section are true if the canonical maps

$$
\zeta_{H, G}: H^{*} \otimes G \rightarrow \operatorname{Hom}(H, G) \quad \text { and } \quad \zeta_{G, F}: G^{*} \otimes F \rightarrow \operatorname{Hom}(G, F)
$$

are isomorphisms. This holds, in particular, if $H$ and $F$ are finitely generated free modules. For simplicity we restrict the presentation to this case.

We start on associating a Koszul complex with the map $\psi$. By virtue of $\zeta_{G, F}$ we may consider $\psi$ an element of $\bigwedge_{S(F)}\left(G^{*} \otimes S(F)\right)$ and, using the canonical isomorphism of $S(F)$-algebras

$$
\bigwedge_{S(F)}\left(G^{*} \otimes S(F)\right) \cong \bigwedge G^{*} \otimes S(F),
$$

an element of $\bigwedge G^{*} \otimes S(F)$.

Let $N$ be a right $\bigwedge G^{*}$-module and $M$ an $S(F)$-module. Then $N \otimes_{R} M$ is a right $\bigwedge G^{*} \otimes S(F)$-module by

$$
(n \otimes m)<\left(y^{*} \otimes f\right)=\left(n \leftarrow y^{*}\right) \otimes f m,
$$

$n \in N, m \in M, y^{*} \in \bigwedge G^{*}, f \in S(F)$. If $M=\oplus_{i} M_{i}$ is a graded $S(F)$-module, then the right multiplication by $\psi$, denoted by $\partial_{\psi}$, maps $N \otimes_{R} M_{i-1}$ to $N \otimes_{R} M_{i}$. Since $\psi^{2}=0$ in $\bigwedge G^{*} \otimes S(F)$, we obtain a complex of $R$-modules

$$
\cdots \rightarrow N \otimes M_{i-1} \stackrel{\partial_{\psi}}{\rightarrow} N \otimes M_{i} \stackrel{\partial_{\psi}}{\rightarrow} N \otimes M_{i+1} \rightarrow \cdots .
$$

If in particular $N=\bigwedge G$ equipped with the $\bigwedge G^{*}$-module structure defined in section 2. then an easy calculation shows that

$$
\partial_{\psi}\left(y_{1} \wedge \ldots \wedge y_{p} \otimes m\right)=\sum_{j=1}^{p}(-1)^{j+1} y_{1} \wedge \ldots \widehat{y_{j}} \ldots \wedge y_{p} \otimes \psi\left(y_{j}\right) m
$$

for all $y_{1}, \ldots, y_{p} \in G$ and $m \in M$.

We are mainly interested in two special cases for $M$, namely $M=S(F)$ where $M_{i}=S_{i}(F)$, and $M=S(F)^{*}$ where $M_{i}=S_{-i}(F)^{*}$.

Let $f_{1}, \ldots, f_{m}$ be a basis of $F$ and $f_{1}^{*}, \ldots, f_{m}^{*}$ the dual basis of $F^{*}$. Then $\psi$, as an element of $\bigwedge G^{*} \otimes S(F)$, has the presentation

$$
\psi=\sum_{j=1}^{m} \psi^{*}\left(f_{j}^{*}\right) \otimes f_{j}
$$

Set $x^{*}=\psi^{*}\left(f_{1}^{*}\right) \wedge \ldots \wedge \psi^{*}\left(f_{m}^{*}\right) \in \bigwedge G^{*}$. We obtain immediately that $\left(x^{*} \otimes 1_{S(F)}\right) \psi=$ $\psi\left(x^{*} \otimes 1_{S(F)}\right)=0$ in $\bigwedge G^{*} \otimes S(F)$. Therefore we may use the right multiplication by 
$x^{*}$ on $\bigwedge G$, which we denote by $\nu_{\psi}$, in order to splice the complexes $\left(\bigwedge G \otimes S(F)^{*}, \partial_{\psi}\right)$ and $\left(\bigwedge G \otimes S(F), \partial_{\psi}\right)$ to a complex

$$
\cdots \rightarrow \bigwedge G \otimes S_{1}(F)^{*} \stackrel{\partial_{\psi}}{\rightarrow} \bigwedge G \otimes S_{0}(F)^{*} \stackrel{\nu_{\psi}}{\rightarrow} \bigwedge G \otimes S_{0}(F) \stackrel{\partial_{\psi}}{\rightarrow} \bigwedge G \otimes S_{1}(F) \rightarrow \cdots .
$$

This complex, as a complex of $R$-modules, splits into direct summands

$$
\begin{array}{r}
\mathcal{C}_{\psi}(t): \quad \cdots \rightarrow \bigwedge^{t+m+p} G \otimes S_{p}(F)^{*} \stackrel{\partial_{\psi}}{\rightarrow} \cdots \stackrel{\partial_{\psi}}{\rightarrow} \bigwedge^{t+m} G \otimes S_{0}(F)^{*} \stackrel{\nu_{\psi}}{\rightarrow} \bigwedge^{t} G \otimes S_{0}(F) \stackrel{\partial_{\psi}}{\rightarrow} \\
\cdots \stackrel{\partial_{\psi}}{\rightarrow} \bigwedge^{0} G \otimes S_{t}(F) \rightarrow 0 .
\end{array}
$$

REMARKS 3.1. (a) Clearly the connection map $\nu_{\psi}$ in $\mathcal{C}_{\psi}(t)$ depends on the basis we took for $F$. To avoid notational complications we ignore this fact, the more so as it doesn't affect the homology.

(b) We specialize to the case in which $F=R$ and $x^{*}=\psi^{*}(1)=\psi$. One can easily check that, for all $t \in \mathbb{Z}, \mathcal{C}_{\psi}(t)$ is the classical Koszul complex associated with $\psi$.

There are well-known generalizations of the classical Koszul complex in case $G$ is a finitely generated free $R$-module, due to Eagon and Northcott, Buchsbaum and $\operatorname{Rim}$, and others. Suppose $G$ to be free of finite rank, and denote by $\mathcal{C}^{t}(\psi)$ the complexes as introduced in [E], A2.6.1 or in [BV2], 2.C. They are constructed as follows. Set rank $G=n$, and consider the direct $(R$-)summands

$$
0 \rightarrow \bigwedge^{n-m-t} G \otimes S_{0}(F) \rightarrow \cdots \stackrel{\partial_{\psi}}{\rightarrow} \bigwedge^{0} G \otimes S_{n-m-t}(F) \rightarrow 0
$$

and

$$
0 \rightarrow \bigwedge^{t} G \otimes S_{0}(F) \rightarrow \cdots \stackrel{\partial_{\psi}}{\rightarrow} \bigwedge^{0} G \otimes S_{t}(F) \rightarrow 0
$$

of $\left(\bigwedge G \otimes S(F), \partial_{\psi}\right)$. We shall connect the $R$-dual of the first complex with the second one. Let $\omega: \bigwedge^{n} G \rightarrow R$ be an orientation of $G$. Then there is a basis $y_{1}, \ldots, y_{n}$ of $G$ such that $\omega\left(y_{1} \wedge \ldots \wedge y_{n}\right)=1$. Denote by $\widetilde{\omega}: \wedge^{n} G^{*} \rightarrow R$ the orientation of $G^{*}$ induced by $\omega$, i.e. $\widetilde{\omega}\left(y_{1}^{*} \wedge \ldots \wedge y_{n}^{*}\right)=1$ where $y_{1}^{*}, \ldots, y_{n}^{*}$ is the basis of $G^{*}$ dual to $y_{1}, \ldots, y_{n}$. Let $x^{*}=\psi^{*}\left(f_{1}^{*}\right) \wedge \ldots \wedge \psi^{*}\left(f_{m}^{*}\right)$ as above, and consider the map

$$
\widetilde{\nu}_{\psi}: \bigwedge^{n-m-t} G^{*} \rightarrow\left(\bigwedge^{t} G^{*}\right)^{*}, \quad \widetilde{\nu}_{\psi}\left(z^{*}\right)\left(y^{*}\right)=\widetilde{\omega}\left(z^{*} \wedge y^{*} \wedge x^{*}\right)
$$

for all $z^{*} \in \bigwedge^{n-m-t} G^{*}, y^{*} \in \bigwedge^{t} G^{*}$. Furthermore let $\mathcal{C}^{t}(\psi)$ be the $R$-sequence

$$
\begin{aligned}
0 \rightarrow\left(\bigwedge^{0} G \otimes S_{r-t}(F)\right)^{*} \stackrel{\partial_{\psi}^{*}}{\rightarrow} \cdots \stackrel{\partial_{\psi}^{*}}{\rightarrow}\left(\bigwedge^{n-m-t} G \otimes S_{0}(F)\right)^{*} & \\
& \stackrel{\widetilde{\nu}_{\psi}}{\rightarrow} \bigwedge^{t} G \otimes S_{0}(F) \stackrel{\partial_{\psi}}{\rightarrow} \cdots \stackrel{\partial_{\psi}}{\rightarrow} \bigwedge^{0} G \otimes S_{t}(F) \rightarrow 0 .
\end{aligned}
$$

Here we set $\left(\bigwedge^{p} G\right)^{*}=\bigwedge^{p} G^{*}$ and $\left(\bigwedge^{t} G^{*}\right)^{*}=\bigwedge^{t} G$ via the corresponding canonical maps. The following proposition shows that $\mathcal{C}^{t}(\psi)$ is a complex, and that there is a complex isomorphism $\mathcal{C}_{\psi}(t) \cong \mathcal{C}^{t}(\psi)$. 
PROPOSITION 3.2. With the assumptions and the notation from above let

$$
\omega_{p}: \bigwedge^{p} G \rightarrow\left(\bigwedge^{n-p} G\right)^{*}, \quad p=0, \ldots, n
$$

be the isomorphisms given by

$$
\left(\omega_{p}(x)\right)(y)=\omega(x \wedge y)
$$

for $x \in \bigwedge^{p} G, y \in \bigwedge^{n-p} G$. Set $S_{p}=S_{p}(F), S_{p}^{*}=S_{p}(F)^{*}, \bigwedge^{p}=\bigwedge^{p} G$. Then the diagram

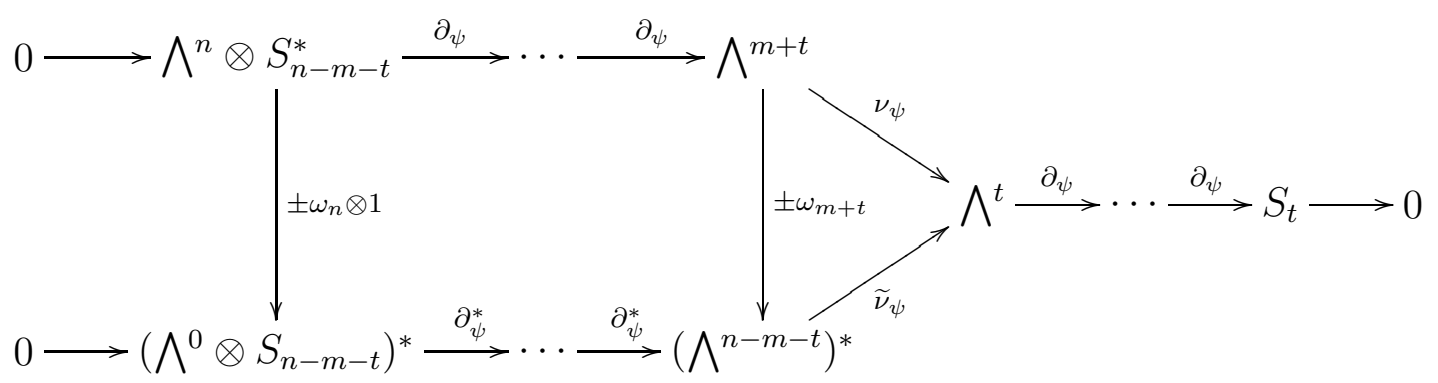

is commutative. In particular there are (non-canonical) complex isomorphisms

$$
\mathcal{C}_{\psi}(t) \cong \mathcal{C}^{t}(\psi)
$$

Proof. We start with showing that the diagram

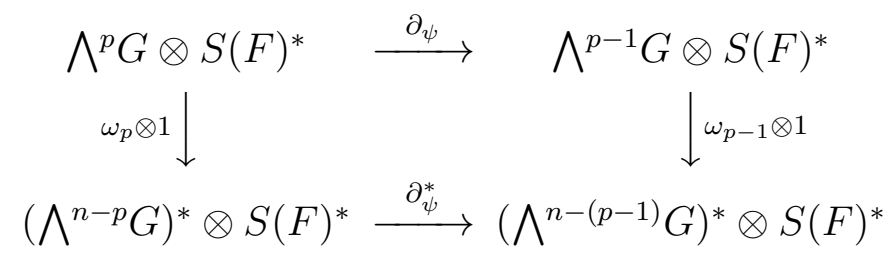

is commutative or anticommutative. Let $x \in \bigwedge^{p} G, y \in \bigwedge^{n-(p-1)} G, f \in S(F)$ and $f^{*} \in S(F)^{*}$. Using formula (2) from above, one easily shows that

$$
\left(\omega_{p-1} \otimes 1\right) \partial_{\psi}\left(x \otimes f^{*}\right)(y \otimes f)=\left(\omega \otimes f^{*}\right)\left(\partial_{\psi}(x \otimes f)(y \otimes 1)\right),
$$

and

$$
\partial_{\psi}^{*}\left(\omega_{p} \otimes 1\right)\left(x \otimes f^{*}\right)(y \otimes f)=\left(\omega \otimes f^{*}\right)\left((x \otimes 1) \partial_{\psi}(y \otimes f)\right) .
$$

Since

$$
0=\partial_{\psi}(x \wedge y \otimes f)=\partial_{\psi}(x \otimes f)(y \otimes 1)+(-1)^{p}(x \otimes 1) \partial_{\psi}(y \otimes f),
$$

by formula (11) in section 2, we are done. 
It remains to prove that the 'triangle' is commutative or anticommutative, that is $\nu_{\psi}= \pm \widetilde{\nu}_{\psi} \omega_{m+t}$. We consider the diagram

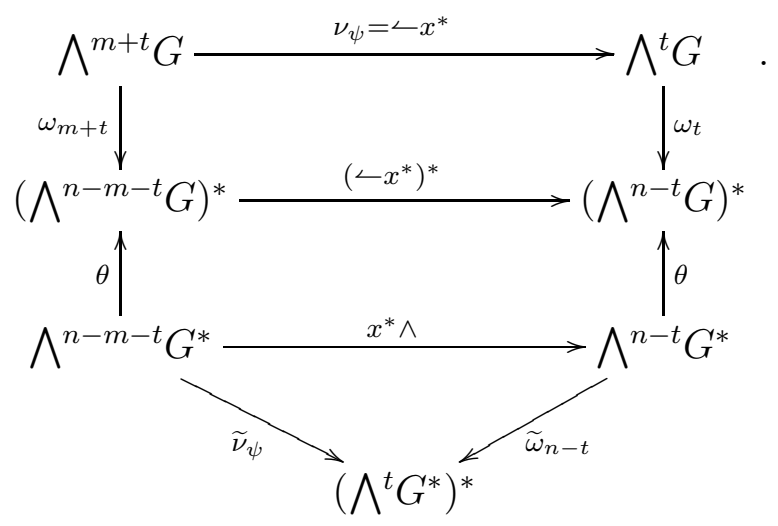

Using formula (11) in section 2 one sees easily that the first rectangle is commutative or anticommutative. The second is commutative by 2.2. Finally

$$
\widetilde{\nu}_{\psi}\left(z^{*}\right)\left(y^{*}\right)=\widetilde{\omega}\left(z^{*} \wedge y^{*} \wedge x^{*}\right)= \pm \widetilde{\omega}\left(x^{*} \wedge z^{*} \wedge y^{*}\right)= \pm \widetilde{\omega}_{n-t}\left(x^{*} \wedge z^{*}\right)\left(y^{*}\right)
$$

for all $z^{*} \in \bigwedge^{n-m-t} G^{*}, y^{*} \in \bigwedge^{n-t} G^{*}$. An equally simple computation shows that $\widetilde{\omega}_{n-t} \theta^{-1} \omega_{t}$ is the canonical map $\bigwedge^{t} G \rightarrow\left(\bigwedge^{t} G^{*}\right)^{*}$.

COROLLARY 3.3. If $G$ is free of rank $n$, then there are (non-canonical) complex isomorphisms

$$
\mathcal{C}_{\psi}(t) \cong\left(\mathcal{C}_{\psi}(n-m-t)\right)^{*}
$$

Proof. Since $\mathcal{C}^{t}(\psi)$ is dual to $\mathcal{C}^{n-m-t}(\psi)$ (see [E], Chapter A.2.6.1, for example), the corollary follows immediately from the preceding proposition.

Now we shall associate a complex with the map $\varphi: H \rightarrow G$, generalizing the dual version of the classical Koszul complex. Since the canonical map $\zeta_{H, G}: H^{*} \otimes G \rightarrow$ $\operatorname{Hom}(H, G)$ is an isomorphism, we may view $\varphi$ as an element of the $S\left(H^{*}\right)$-algebra

$$
S\left(H^{*}\right) \otimes \bigwedge G=\bigwedge_{S\left(H^{*}\right)}\left(S\left(H^{*}\right) \otimes G\right) .
$$

We have $\varphi^{2}=0$, so for every graded $S\left(H^{*}\right)$-module $M=\oplus_{i} M_{i}$, the left multiplication by $\varphi$ on $M \otimes \wedge G$, denoted by $d_{\varphi}$, gives rise to a complex of $\bigwedge G$-modules

$$
\cdots \rightarrow M_{i-1} \otimes \bigwedge G \stackrel{d_{\varphi}}{\rightarrow} M_{i} \otimes \bigwedge G \stackrel{d_{\varphi}}{\rightarrow} M_{i+1} \otimes \bigwedge G \rightarrow \cdots .
$$

Two cases for $M$ lie in our interest: $M=S\left(H^{*}\right)$ with the natural grading, and $M=D(H)$ where the structure of $D(H)$ as a graded $S\left(H^{*}\right)$-module has been described in section 2 and $M_{i}=D_{-i}(H)$ in this case. An easy calculation shows that

$$
d_{\varphi}\left(h_{1}^{\left(k_{1}\right)} \ldots h_{q}^{\left(k_{q}\right)} \otimes y\right)=\sum_{j=1}^{q} h_{1}^{\left(k_{1}\right)} \ldots h_{j}^{\left(k_{j}-1\right)} \ldots h_{q}^{\left(k_{q}\right)} \otimes \varphi\left(h_{j}\right) \wedge y
$$


for all $h_{1}, \ldots, h_{q} \in H, y \in \bigwedge G$.

Let $h_{1}, \ldots, h_{l}$ be a basis of $H, h_{1}^{*}, \ldots, h_{l}^{*}$ the dual basis of $H^{*}$, and set $x=$ $\varphi\left(h_{1}\right) \wedge \ldots \wedge \varphi\left(h_{l}\right) \in \wedge G$. Then $\varphi$, as an element of $S\left(H^{*}\right) \otimes \wedge G$, has the presentation

$$
\varphi=\sum_{j=1}^{l} h_{j}^{*} \otimes \varphi\left(h_{j}\right),
$$

consequently $\left(1_{S\left(H^{*}\right)} \otimes x\right) \varphi=\varphi\left(1_{S\left(H^{*}\right)} \otimes x\right)=0$ in $S\left(H^{*}\right) \otimes \bigwedge G$. So we may use the left multiplication by $x$ on $\bigwedge G$, denoted by $\nu^{\varphi}$, in order to splice the complexes $\left(D(H) \otimes \bigwedge G, d_{\varphi}\right)$ and $\left(S\left(H^{*}\right) \otimes \bigwedge G, d_{\varphi}\right)$ to a complex

$$
\cdots \rightarrow D_{1}(H) \otimes \bigwedge G \stackrel{d_{\varphi}}{\rightarrow} D_{0}(H) \otimes \bigwedge G \stackrel{\nu^{\varphi}}{\rightarrow} S_{0}\left(H^{*}\right) \otimes \bigwedge G \stackrel{d_{\varphi}}{\rightarrow} S_{1}\left(H^{*}\right) \otimes \bigwedge G \rightarrow \cdots .
$$

This complex, as a complex of $R$-modules, splits into direct summands

$$
\begin{aligned}
\mathcal{D}_{\varphi}(t): \quad 0 \rightarrow D_{t}(H) \otimes \bigwedge^{0} G \stackrel{d_{\varphi}}{\rightarrow} \cdots \stackrel{d_{\varphi}}{\rightarrow} D_{0}(H) \otimes \bigwedge^{t} G \stackrel{\nu^{\varphi}}{\rightarrow} S_{0}\left(H^{*}\right) \otimes \bigwedge^{t+l} G \stackrel{d_{\varphi}}{\rightarrow} \cdots \\
\stackrel{d_{\varphi}}{\rightarrow} S_{p}\left(H^{*}\right) \otimes \bigwedge^{t+l+p} G \rightarrow \cdots
\end{aligned}
$$

Similarly as in the case of $\mathcal{C}_{\psi}(t)$ (see remark 3.1), the connection map $\nu^{\varphi}$ in $\mathcal{D}_{\varphi}(t)$ depends on the basis taken for $H$. Moreover there is an analogue with Proposition 3.2 and its proof.

PROPOSITION 3.4. Suppose $G$ to be a free $R$-module of rank $n$. Then $\mathcal{D}_{\varphi}(t)$ is (non-canonically) isomorphic to the complex $\mathcal{D}^{t}(\varphi)$ as defined in [BV1].

We are now able to formulate a general result concerning the connection of the generalized Koszul complex and its dual through natural complex isomorphisms.

PROPOSITION 3.5. Let $\psi: G \rightarrow F$ be a homomorphism of $R$-modules where $F$ is free of finite rank. Then the canonical map $\theta: \bigwedge G^{*} \rightarrow(\bigwedge G)^{*}$ induces a natural complex morphism

$$
\tau: \mathcal{D}_{\psi *}(t) \rightarrow\left(\mathcal{C}_{\psi}(t)\right)^{*}
$$

where the connection homomorphisms $\nu^{\psi^{*}}$ and $\nu_{\psi}$ are defined with respect to the same basis of $F$. If $\theta$ is an isomorphism (for example, if $G$ is finitely generated and projective), then $\tau$ is a complex isomorphism.

Proof. We identify $D\left(F^{*}\right)=S(F)^{*}$ and $S(F)=S(F)^{* *}$ by the corresponding graded algebra isomorphisms. Then we define $\tau: D\left(F^{*}\right) \otimes \bigwedge G^{*} \rightarrow(\bigwedge G)^{*} \otimes S(F)^{*}$ as the composition of the canonical map $D\left(F^{*}\right) \otimes \bigwedge G^{*} \rightarrow \bigwedge G^{*} \otimes S(F)^{*}$ and $\theta \otimes 1_{S(F)^{*} \text {. }}$ The map $\tau: S(F) \otimes \bigwedge^{*} \rightarrow(\bigwedge G)^{*} \otimes S(F)^{* *}$ is defined analogously.

To show that $\tau$ is a complex morphism, we choose a basis $f_{1}, \ldots, f_{m}$ for $F$. Let $f_{1}^{*}, \ldots, f_{m}^{*}$ be the dual basis of $F^{*}$. Then $\psi^{*}=\sum_{j} f_{j} \otimes \psi^{*}\left(f_{j}^{*}\right)$ as an element of $F \otimes G^{*}$, and

$$
d_{\psi^{*}}=\sum_{j} f_{j} 1_{A} \otimes\left(\psi^{*}\left(f_{j}^{*}\right) \wedge\right) \quad \text { and } \quad\left(\partial_{\psi}\right)^{*}=\sum_{j}\left(\left\llcorner\psi^{*}\left(f_{j}^{*}\right)\right)^{*} \otimes f_{j} 1_{A}\right.
$$


where $A$ stands for $D\left(F^{*}\right), S(F), S(F)^{*}, S(F)^{* *}$ respectively. Similarly

$$
\nu^{\psi^{*}}=\psi^{*}\left(f_{1}^{*}\right) \wedge \ldots \wedge \psi^{*}\left(f_{m}^{*}\right) \wedge \quad \text { and } \quad\left(\nu_{\psi}\right)^{*}=\left(\left\llcorner\psi^{*}\left(f_{1}^{*}\right) \wedge \ldots \wedge \psi^{*}\left(f_{m}^{*}\right)\right)^{*} .\right.
$$

An application of Proposition 2.2 now finishes the proof.

REMARK 3.6. Suppose $G$ to be finitely generated free. Using the above result we get natural isomorphisms

$$
\mathcal{C}_{\psi}(t) \cong\left(\mathcal{C}_{\psi}(t)\right)^{* *} \cong\left(\mathcal{D}_{\psi *}(t)\right)^{*}
$$

So we may canonically identify $\mathcal{C}_{\psi}(t)$ with the generic resolution introduced by Buchsbaum and Eisenbud in [BE3].

\section{Koszul Bicomplexes}

Let $F, G, H$ be $R$-modules. We assume $H$ and $F$ to be free of ranks $l$ and $m$. Let

$$
H \stackrel{\varphi}{\longrightarrow} G \stackrel{\psi}{\longrightarrow} F
$$

be a complex. Due to Proposition 2.1 we can assemble the complexes $\mathcal{C}_{\psi}(t)$ and $\mathcal{D}_{\varphi}(t)$ to our main tool, the bicomplexes $\mathcal{K}_{., .}(t)$

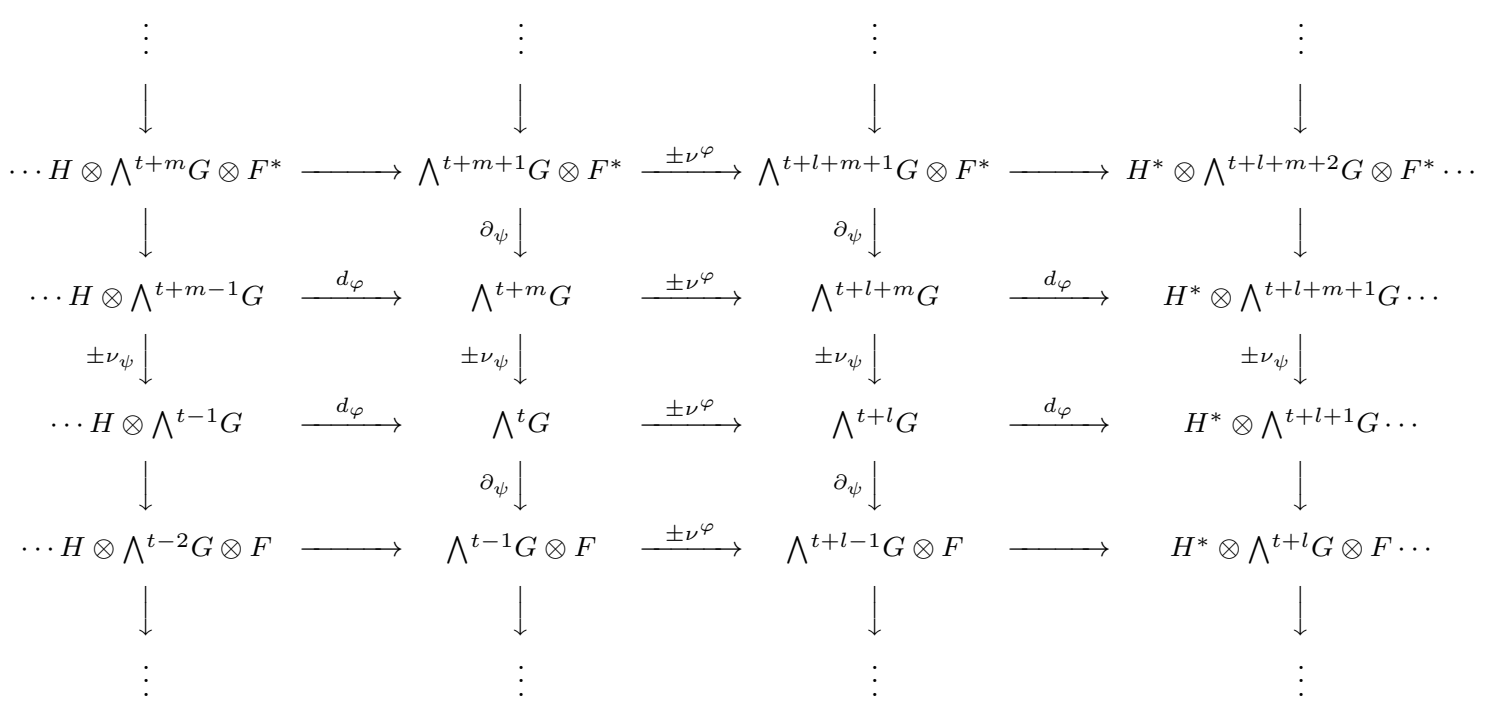

The rows in the upper half are built from $\mathcal{D}_{\varphi}(t+m+j)$ tensored with $S_{j}(F)^{*}$, $j=0,1, \ldots$, while the rows below are built from $\mathcal{D}_{\varphi}(t-j)$ tensored with $S_{j}(F)$, $j=0,1, \ldots$; we abbreviate $d_{\varphi} \otimes 1_{S\left(F^{*}\right)}$ and $d_{\varphi} \otimes 1_{S(F)}$ to $d_{\varphi}$, and correspondingly $\nu^{\varphi} \otimes 1_{S\left(F^{*}\right)}$ and $\nu^{\varphi} \otimes 1_{S(F)}$ to $\nu^{\varphi}$. The columns are obtained analogously: in western direction we have to tensorize $D_{i}(H)$ with $\mathcal{C}_{\psi}(t-i), i=0,1, \ldots$, while going east we must tensorize $S_{i}\left(H^{*}\right)$ with $\mathcal{C}_{\psi}(t+l+i), i=0,1, \ldots$; as before we shorten the 
complex maps to $\partial_{\psi}$ and $\nu_{\psi}$. The signs of $\nu^{\varphi}$ and $\nu_{\psi}$ are determined by the formula in Proposition 2.1.

To prove that the rectangles in $\mathcal{K}_{., .}(t)$ are anticommutative, is an easy exercise. We show for example that $\partial_{\psi} d_{\varphi}+d_{\varphi} \partial_{\psi}=0$.

Let $M$ be a graded $S\left(H^{*}\right)$-module and $N$ a graded $S(F)$-module. Then the diagram

$$
\begin{array}{cr}
M \otimes \bigwedge G \otimes N \stackrel{d_{\varphi} \otimes 1_{N}}{\longrightarrow} M \otimes \bigwedge G \otimes N \\
1_{M} \otimes \partial_{\psi} \downarrow & \left\lfloor 1_{M} \otimes \partial_{\psi}\right. \\
M \otimes \bigwedge G \otimes N \stackrel{d_{\varphi} \otimes 1_{N}}{\longrightarrow} M \otimes \bigwedge G \otimes N .
\end{array}
$$

is anticommutative: as above let $h_{1}, \ldots, h_{l}$ and $f_{1}, \ldots, f_{m}$ be bases of $H$ and $F$, and denote by $h_{1}^{*}, \ldots, h_{l}^{*}$ and $f_{1}^{*}, \ldots, f_{m}^{*}$ the corresponding dual bases. Then $\varphi=$ $\sum_{i} h_{i}^{*} \otimes \varphi\left(h_{i}\right)$ and $\psi=\sum_{j} \psi^{*}\left(f_{j}^{*}\right) \otimes f_{j}$ as elements of $H^{*} \otimes G$ and $G^{*} \otimes F$ respectively. Then for $m \in M, y \in \bigwedge G$, and $n \in N$

$$
\left(1_{M} \otimes \partial_{\psi}\right)\left(d_{\varphi} \otimes 1_{N}\right)(m \otimes y \otimes n)=\sum_{i} \sum_{j} h_{i}^{*} m \otimes\left(\left(\varphi\left(h_{i}\right) \wedge y\right) \leftarrow \psi^{*}\left(f_{j}^{*}\right)\right) \otimes f_{j} n
$$

while

$$
\left(d_{\varphi} \otimes 1_{N}\right)\left(1_{M} \otimes \partial_{\psi}\right)(m \otimes y \otimes n)=\sum_{j} \sum_{i} h_{i}^{*} m \otimes\left(\varphi\left(h_{i}\right) \wedge\left(y \leftarrow \psi^{*}\left(f_{j}^{*}\right)\right)\right) \otimes f_{j} n .
$$

Since $\psi^{*}\left(f_{j}^{*}\right)\left(\varphi\left(h_{i}\right)\right)=f_{j}^{*} \psi \varphi\left(h_{i}\right)=0$, the associativity formula of Proposition 2.1 yields the desired result.

\section{Grade Sensitivity}

This section links up the study of the Koszul bicomplexes introduced in the previous section with the study of the homology of certain Koszul complexes. To avoid permanent repetitions we shall assume throughout that the $\operatorname{ring} R$ is noetherian.

For a linear map $\varphi: H \rightarrow G$ of finitely generated free $R$-modules we denote by $I_{\varphi}$ the ideal in $R$ generated by the maximal minors of a matrix representing $\varphi$. We shall use Macaulay's Theorem several times which says that $\operatorname{grade} I_{\varphi} \leq$ $|\operatorname{rank} G-\operatorname{rank} H|+1$ if $I_{\varphi} \neq R$.

Our general assumption throughout the rest of this section will be that $H, G$ and $F$ are free $R$-modules of finite ranks $l, n$ and $m$, and that

$$
H \stackrel{\varphi}{\longrightarrow} G \stackrel{\psi}{\longrightarrow} F
$$

is a complex. Although much of what we will do, holds formally for any $l, n$ and $m$, the applications will refer to the case in which $n \geq m$ and $n \geq l$. So we suppose that $r=n-m \geq 0, s=n-l \geq 0$. We set $g=\operatorname{grade} I_{\psi}$ and $h=\operatorname{grade} I_{\varphi}$. 
A first question is which restrictions $g$ and $h$ are subjected in a situation as the one pictured above. In the sequel we shall give some answers to this question. The following result is a simple consequence of Macaulay's Theorem.

PROPOSITION 5.1. Set $\rho=r-l$.

(1) If $g, h \geq 1$, then $\rho \geq 0$.

(2) If $g>|\rho|+1$, then $I_{\varphi} \subset \operatorname{Rad} I_{\psi}$, and in particular $h \leq g$.

(3) Moreover, if $g \geq r+1$, then $I_{\varphi} \subset I_{\psi}$.

Proof. If $g \geq 1$, then $M=\operatorname{Coker} \psi^{*}$ has $\operatorname{rank} r$. So $M^{*}=\operatorname{Ker} \psi$ has $\operatorname{rank} r$, too. In the same way $h \geq 1$ implies that $\varphi$ is injective, so $\operatorname{Im} \varphi$ has $\operatorname{rank} l$. Since $\operatorname{Ker} \psi \supset \operatorname{Im} \varphi$, we obtain the first part.

While proving (2) and (3) we may assume that $I_{\psi} \neq R$. Suppose that $g>$ $|\rho|+1$. Take a prime ideal $P \supset I_{\psi}$ in $R$. Then grade $\left(I_{\psi} R_{P}\right) \geq g$. If $I_{\varphi} \not \subset P$, then $(\operatorname{Im} \varphi)_{P}$ would be a free direct summand of $G_{P}$ of rank $l$, and therefore $\psi_{P}$ can be viewed as a map from a free module of rank $n-l$ to a free module of rank $m$. So grade $\left(I_{\psi} R_{P}\right) \leq|\rho|+1$, which contradicts the hypothesis. It follows that $I_{\varphi} \subset P$ which implies that $I_{\varphi} \subset \operatorname{Rad} I_{\psi}$.

In case $g=\operatorname{grade} I_{\psi}=r+1$, we consider a rank 1 direct summand $\widetilde{H}$ of $H$. Let $\widetilde{\varphi}$ be the restriction of $\varphi$ to $\widetilde{H}$. From [BV4], Proposition 1, we draw that $I_{\widetilde{\varphi}} \subset I_{\psi}$ in this case. Since $I_{\varphi} \subset I_{\tilde{\varphi}}$, the conclusion follows.

Since $G$ is finitely generated, the generalized Koszul complexes $\mathcal{C}_{\psi}(t)$ and $\mathcal{D}_{\varphi}(t)$ (see section 31) have only a finite number of non-vanishing components. To identify the homology, we fix their graduations as follows: position 0 is held by the leftmost non-zero module. The homology of $\mathcal{C}_{\psi}(t)$ and $\mathcal{D}_{\varphi}(t)$ behaves similarly as the homology of the classical Koszul complex. The main result is the following.

THEOREM 5.2. Set $C=$ Coker $\psi$ and $D=$ Coker $\varphi^{*}$. Furthermore let $S_{0}(D)=$ $R / I_{\varphi}, S_{-1}(D)=\bigwedge^{s+1}$ Coker $\varphi, S_{0}(C)=R / I_{\psi}$ and $S_{-1}(C)=\bigwedge^{r+1}$ Coker $\psi^{*}$. Then the following hold.

(a) $H^{i}\left(\mathcal{D}_{\varphi}(t)\right)=0$ for $i<h$. Moreover, if $t \leq s+1$ and grade $I_{k}(\varphi) \geq n-k+1$ for all $k$ with $l \geq k \geq 1$, then $\mathcal{D}_{\varphi}(t)$ is a free resolution of $S_{s-t}(D)$. (If $-1 \leq t \leq s+1$, then it suffices to require that grade $I_{\varphi} \geq s+1$.)

(b) $H^{i}\left(\mathcal{C}_{\psi}(t)\right)=0$ for $i<g$. Moreover, if $t \geq-1$ and grade $I_{k}(\psi) \geq n-k+1$ for all $k$ with $m \geq k \geq 1$, then $\mathcal{C}_{\psi}(t)$ is a free resolution of $S_{t}(C)$. (If $-1 \leq t \leq r+1$, then it suffices to require that grade $I_{\psi} \geq r+1$.)

Finally, if $I_{\varphi}=R\left(I_{\psi}=R\right)$, then all sequences $\mathcal{D}_{\varphi}(t)\left(\mathcal{C}_{\psi}(t)\right)$ are split exact. 
Proof. Proposition 3.4. Proposition 3.5 and Corollary 3.3 provide complex isomorphisms

$$
\mathcal{D}^{t}(\varphi) \cong \mathcal{D}_{\varphi}(t) \cong \mathcal{C}_{\varphi^{*}}(s-t)
$$

and similarly we obtain

$$
\mathcal{C}^{t}(\psi) \cong \mathcal{C}_{\psi}(t) \cong \mathcal{D}_{\psi^{*}}(r-t) .
$$

Since grade $I_{\varphi}=\operatorname{grade} I_{\varphi^{*}}$ and grade $I_{\psi}=\operatorname{grade} I_{\psi^{*}}$, (a) follows from Proposition 2.1 in [BV1] while (b) is obtained from Theorem A2.10,(c) in [E].

By $\mathcal{C}_{., .}(t)$ we shall denote the bicomplex which is the lower part of the Koszul bicomplex in the previous section (the rows below the second row). We rewrite this complex as

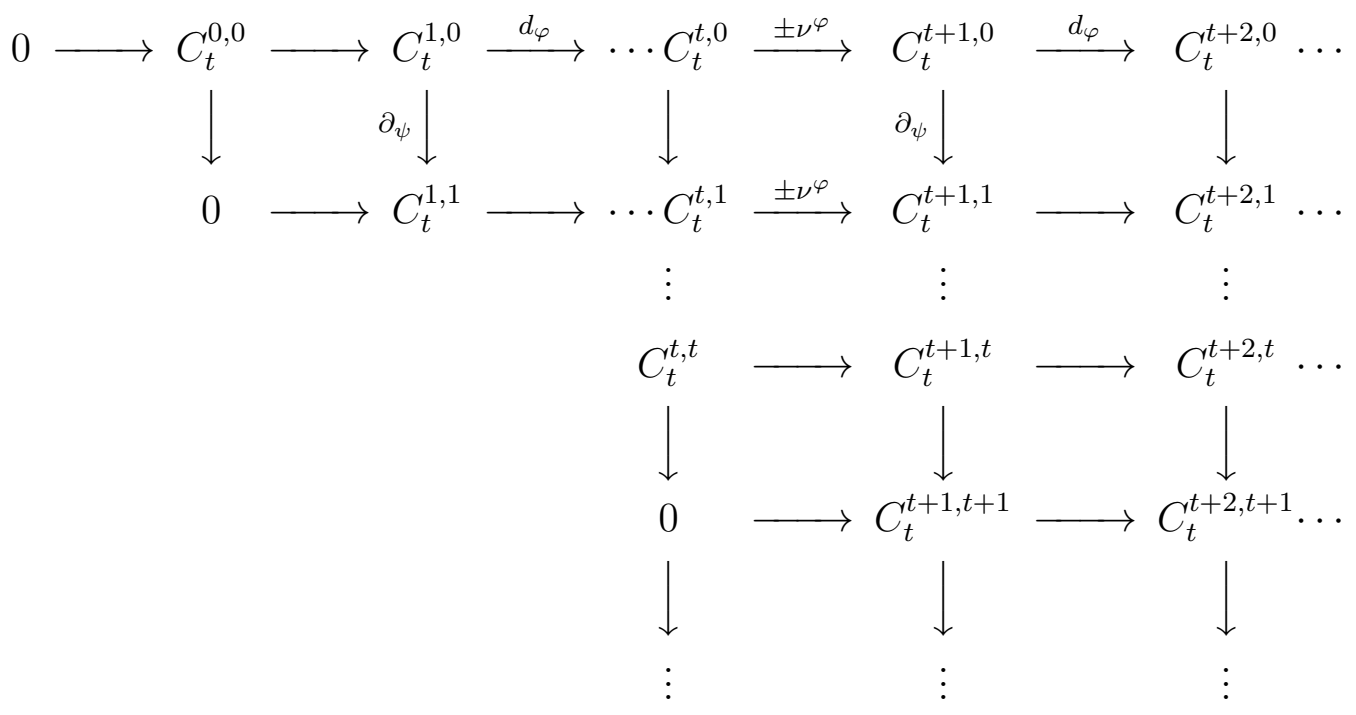

In other words,

$$
C_{t}^{0,0}= \begin{cases}D_{t}(H) \otimes \bigwedge^{0} G \otimes S_{0}(F) & \text { if } \quad 0 \leq t, \\ S_{0}\left(H^{*}\right) \otimes \bigwedge^{t+l} G \otimes S_{0}(F) & \text { if } \quad-l \leq t<0, \\ S_{-t-l}\left(H^{*}\right) \otimes \bigwedge^{0} G \otimes S_{0}(F) & \text { if } \quad t<-l .\end{cases}
$$

The row homology of $\mathcal{C}_{., .}(t)$ at $C_{t}^{p, q}$ is denoted by $H_{\varphi}^{p, q}$, the column homology by $H_{\psi}^{p, q}$. Thus $H_{\varphi}^{p, 0}$ is the $p$-th homology module of $\mathcal{D}_{\varphi}(t)$.

Set $N^{p}=\operatorname{Ker}\left(C_{t}^{p, 0} \stackrel{\partial_{\psi}}{\rightarrow} C_{t}^{p, 1}\right)$. The canonical injections $N^{p} \rightarrow C_{t}^{p, 0}$ yield a complex morphism

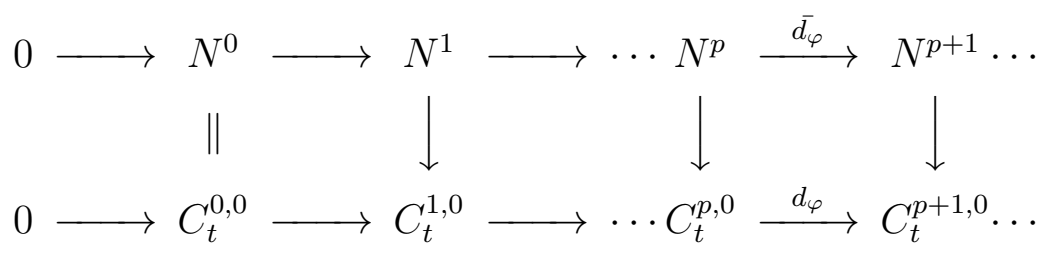


where the maps $\bar{d}_{\varphi}$ are induced by $d_{\varphi}$. The homology of the first row $\mathcal{N}(t)$ at $N^{p}$ is denoted by $\bar{H}^{p}$. We shall now investigate this homology.

For this purpose we extend $\mathcal{C}_{., .}(t)$ to the complex $\widetilde{\mathcal{C}}_{., .}(t)$ by setting $C_{t}^{p,-1}=N^{p}$. We record some facts about the homology of $\widetilde{\mathcal{C}}_{.,}(t)$. To avoid new symbols, the column homology at $C_{t}^{p, q}$ is again denoted by $H_{w}^{p, q}$ (actually it differs from that of $\mathcal{C}_{\text {.,. }}(t)$ only at $C^{p, 0}$ ). We draw from Theorem [5.2, (a) that

$$
H_{\varphi}^{p, q}=0 \quad \text { for } p<h \text { and } q \neq-1 .
$$

In case $r+1-g<p \leq t$, we get from Theorem 5.2 (b)

$$
H_{\psi}^{p, q}=\left\{\begin{array}{cl}
0 & \text { for } 0 \leq q<\min (p-(r+1-g), g), \\
D_{t-p}(H) \otimes S_{p}(C) & \text { for } q=p,
\end{array}\right.
$$

and if $0 \leq t<p$, we obtain

$$
H_{\psi}^{p, q}=\left\{\begin{array}{cl}
0 & \text { for } 0 \leq q<\min (p-(\rho+2-g), g), \\
S_{p-t-1}\left(H^{*}\right) \otimes S_{p+l-1}(C) & \text { for } q=p+l-1 .
\end{array}\right.
$$

For $q \geq-1$ we consider the $q$ th row $\widetilde{C}_{., q}$ of $\widetilde{C}_{., .}$and its image complex $\partial_{\psi}\left(\widetilde{C}_{., q}\right)$ in $\widetilde{C}_{., q+1}$. We set $E^{p, q}=H^{p}\left(\partial_{\psi}\left(\widetilde{C}_{., q-1}\right)\right)$ for $q \geq 0$. Then there are exact sequences

$$
H_{\varphi}^{i-(j+1), j} \rightarrow E^{i-(j+1), j+1} \rightarrow E^{i-j, j} \rightarrow H_{\varphi}^{i-j, j}
$$

if $H_{\psi}^{i-(j+1), j}=H_{\psi}^{i-j, j}=0$, and because of (4) and (5) this holds if

$$
\begin{aligned}
r+1-g & <i-(j+1) \leq t \text { and } j<\min (i-(j+1)-(r+1-g), g) \text { or } \\
0 & \leq t<i-(j+1) \text { and } j<\min (i-(j+1)-(\rho+2-g), g) .
\end{aligned}
$$

THEOREM 5.3. Let $t \geq 0$. Set $C=$ Coker $\psi, \rho=r-l$. Then $\bar{H}^{i}=0$ for $i=0, \ldots, \min (2, h-1)$. Suppose that $g>|\rho|+1$.

(i) If $3 \leq 2 t+1 \leq h$, then there is an injection $D_{0}(H) \otimes S_{t}(C) \rightarrow \bar{H}^{2 t+1}$ which is an isomorphism if $2 t+1<h$;

(ii) $\bar{H}^{i}=0$ for $2 t+2 \leq i<\min (h, 2 t+g-|\rho|+1)$;

(iii) if $2 t+g-|\rho|+1<h$, then $\bar{H}^{2 t+g-|\rho|+1}=H_{\psi}^{t+1, t+g-|\rho|-1}$.

Proof. We may obviously assume that $h>0$. From Proposition 5.1 we then obtain that $\rho \geq 0$. Set $\mu=\min (2, h-1)$. Consider the diagram

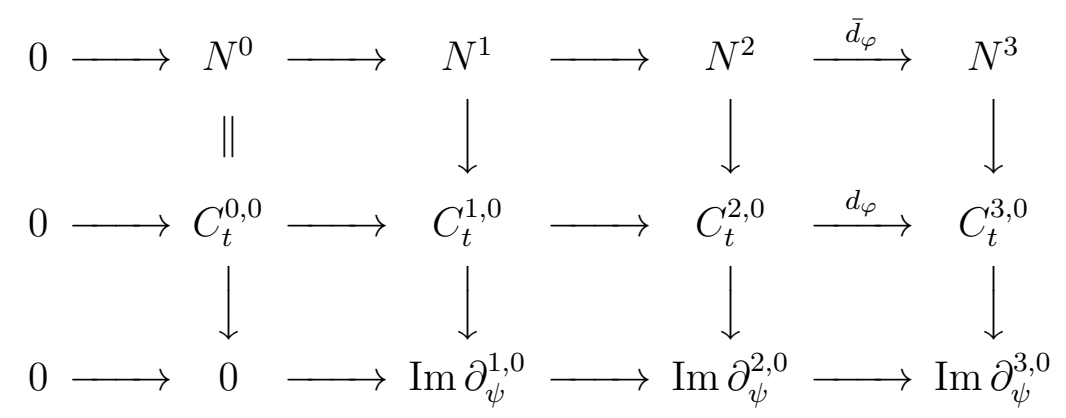


The middle row has trivial homology at $C_{t}^{p, 0}$ for $p \leq \mu$ (Theorem [5.2). Because of $h \geq 1$, the row homology at $\operatorname{Im} \partial_{\psi}^{1,0}$ is zero since the homomorphism $\operatorname{Im} \partial_{\psi}^{1,0} \rightarrow \operatorname{Im} \partial_{\psi}^{2,0}$ is the restriction of the injective homomorphism $C_{t}^{1,1} \stackrel{d_{\varphi}}{\rightarrow} C_{t}^{2,1}$. Now we use the long exact homology sequence to get the first statement.

Next let $g>\rho+1$ and $3 \leq 2 t+1 \leq h$. Remark that $h \leq g$ by Proposition 5.1. (2). Since $H_{\psi}^{2 t-j, j}=H_{\psi}^{2 t+1-j, j}=0$ for $j=0, \ldots, t-1$, we obtain exact sequences

$$
H_{\varphi}^{2 t-j, j} \rightarrow E^{2 t-j, j+1} \rightarrow E^{2 t+1-j, j} \rightarrow H_{\varphi}^{2 t+1-j, j}
$$

for these $j$. Furthermore $2 t+1 \leq h$ by assumption. Therefore we obtain the "southwest" isomorphisms

$$
E^{2 t, 1} \cong \cdots \cong E^{t+2, t-1} \cong E^{t+1, t}
$$

and an injection $E^{2 t, 1} \rightarrow E^{2 t+1,0}=\bar{H}^{2 t+1}$ which is an isomorphism if even $2 t+1<h$. In the diagram

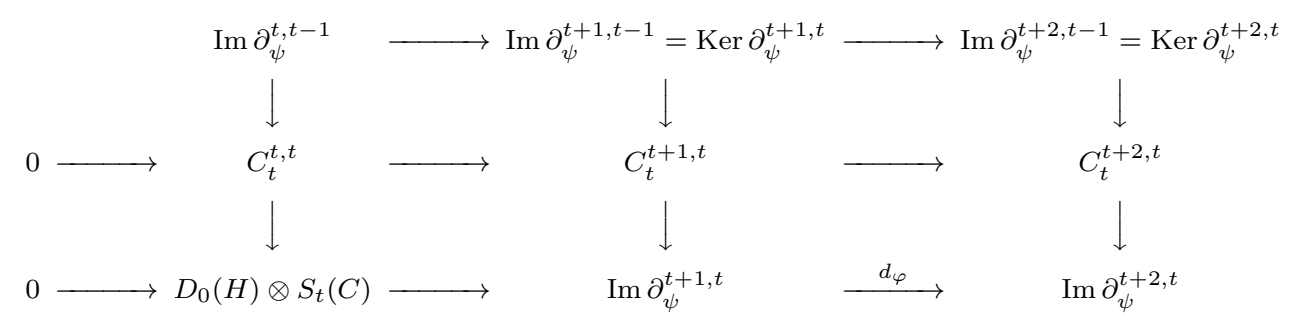

we abbreviate $\partial_{\psi}^{p, q}=\left(C_{t}^{p, q} \stackrel{\partial_{\psi}}{\rightarrow} C_{t}^{p, q+1}\right)$ and $d_{\varphi}^{p, q}=\left(C_{t}^{p, q} \stackrel{d_{\varphi}}{\rightarrow} C_{t}^{p+1, q}\right)$. It is induced by $\mathcal{C}_{. .,}(t)$ and has exact middle row. Since $d_{\varphi}^{t+1, t+1}$ is injective, we obtain $D_{0}(H) \otimes$ $S_{t}(C) \cong E^{t+1, t}$. This together with the considerations in the previous paragraph proves (i).

To prove (ii), let $2 t+2 \leq i<\min (h, 2 t+g-\rho+1)$. Going "southwest" once more, we obtain isomorphisms

$$
\bar{H}^{i}=E^{i, 0} \cong E^{i-1,1} \cong \cdots \cong E^{t+1, i-t-1} .
$$

Since $d_{\varphi}^{t+1, i-t-1}$ is injective, the claim follows.

In case $2 t+g-\rho+1<h$, we get

$$
\bar{H}^{2 t+g-\rho+1}=E^{2 t+g-\rho+1,0} \cong E^{2 t+g-\rho, 1} \cong \ldots \cong E^{t+2, t+g-\rho-1} .
$$

Furthermore the diagram

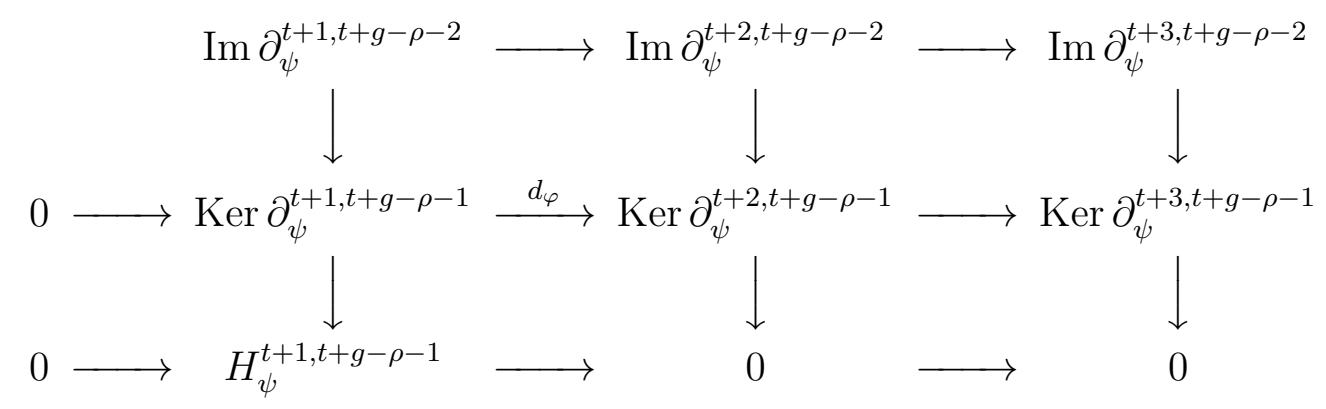


has row homology zero at $\operatorname{Ker} \partial_{\psi}^{t+1, t+g-\rho-1}$ since $d_{\varphi}^{t+1, t+g-\rho-1}$ is injective. If we can show that the row homology at $\operatorname{Ker} \partial_{\psi}^{t+2, t+g-\rho-1}$ also vanishes, we shall obtain (iii). The diagram

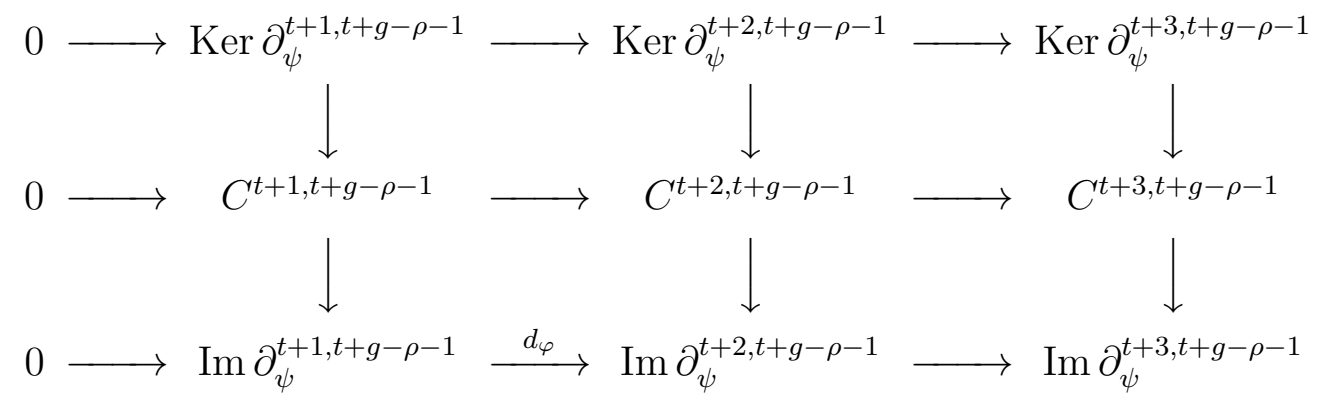

has exact middle row. Since $d_{\varphi}^{t+1, t+g-\rho}$ is injective, we get the desired result.

Provided $g \geq r+1$, we can compute the homology $\bar{H}^{i}$ of $\mathcal{N}(t)$ outside the gap $2 t+1 \leq i<\min (h, 2 t+3+l)$.

THEOREM 5.4. As before let $t \geq 0$, and set $C=$ Coker $\psi, \rho=r-l$. Suppose that $g \geq r+1$. Then

(i)

$$
\bar{H}^{i}=\left\{\begin{array}{cl}
D_{t-\frac{i-1}{2}}(H) \otimes S_{\frac{i-1}{2}}(C) & \text { if } 3 \leq i<\min (h, 2 t+3), i \neq \equiv 0(2) \\
0 & \text { if } 3 \leq i<\min (h, 2 t+3), i \equiv 0(2)
\end{array}\right.
$$

(ii)

$$
\bar{H}^{i}=\left\{\begin{array}{cl}
S_{\frac{i-l}{2}-t-1}(H) \otimes S_{\frac{i+l}{2}-1}(C) & \text { if } 2(t+1)+l \leq i<h, i \equiv l(2), \\
0 & \text { if } 2(t+1)+l \leq i<h, i \neq l(2) .
\end{array}\right.
$$

Proof. For (i) let $3 \leq i<\min (h, 2 t+3)$. Then

$$
H_{\psi}^{i-(j+1), j}=H_{\psi}^{i-j, j}=0 \quad \text { for } \quad \begin{cases}j=0, \ldots, \frac{i-2}{2} & \text { if } i \equiv 0(2) \\ j=0, \ldots, \frac{i-3}{2} & \text { if } i \neq \equiv(2)\end{cases}
$$

Since $H_{\varphi}^{i-(j+1), j}=H_{\varphi}^{i-j, j}=0$ because of $i<h$, the sequences (6) may be used to obtain the isomorphisms

$$
\bar{H}^{i}=E^{i, 0} \cong E^{i-1,1} \cong \cdots \cong E^{\frac{i}{2}, \frac{i}{2}}
$$

if $i$ is even, and

$$
\bar{H}^{i}=E^{i, 0} \cong E^{i-1,1} \cong \ldots \cong E^{\frac{i+1}{2}, \frac{i-1}{2}}
$$

if $i$ is odd. 
In the last case we substitute $t$ by $\frac{i-1}{2}$ and $D_{0}(H) \otimes S_{t}(C)$ by $D_{t-\frac{i-1}{2}} \otimes S_{\frac{i-1}{2}}(C)$ in the second diagram in the proof of the previous theorem. The resulting diagram has the same properties as the original one. Therefore

$$
D_{t-\frac{i-1}{2}} \otimes S_{\frac{i-1}{2}}(C) \cong E^{\frac{i+1}{2}, \frac{i-1}{2}} .
$$

If $i$ is even, we look at the sequence

$$
\operatorname{Im} \partial_{\psi}^{\frac{i-2}{2}, \frac{i-2}{2}} \rightarrow \operatorname{Im} \partial_{\psi}^{\frac{i}{2}, \frac{i-2}{2}} \rightarrow \operatorname{Im} \partial_{\psi}^{\frac{i+2}{2}, \frac{i-2}{2}} .
$$

But $\partial_{\psi}^{\frac{i-2}{2}, \frac{i-2}{2}}=0$, and the second map is induced by the injective map $d_{\varphi}: C^{\frac{i}{2}, \frac{i}{2}} \rightarrow$ $C^{\frac{i+2}{2}, \frac{i}{2}}$. Therefore $E^{\frac{i}{2}, \frac{i}{2}}=0$.

To prove (ii) one uses the same strategy.

REMARKS 5.5. The proofs of Theorems 5.3 and 5.4 partially consist of a repetition of arguments used in the proof of Proposition 1 in [BV4].

One can extend the assertions of 5.3 and 5.4 to negative integers $t$. We touch briefly upon this case omitting the very similar proofs.

THEOREM 5.6. Let $t<0$ be an integer. As above we set $C=$ Coker $\psi, \rho=r-l$.

(a) Suppose that $g>|\rho|+1$. Then

(i) $\bar{H}^{i}=0$ for $0 \leq i<\min (h, \max (2, t+g-|\rho|))$ and

(ii) $\bar{H}^{t+g-|\rho|}=H_{\psi}^{0, t+g-|\rho|-1}$ if $2 \leq t+g-|\rho|<h$.

(b) Suppose that $g \geq r+1$.

(i) If $-l<t$, then

$$
\bar{H}^{i}=\left\{\begin{array}{cl}
S_{\frac{i-l-t-1}{2}}\left(H^{*}\right) \otimes S_{\frac{i+l+t-1}{2}}(C) & \text { if } l+t+1 \leq i<h, i+l+t \not \equiv 0(2), \\
0 & \text { otherwise if } 0 \leq i<h .
\end{array}\right.
$$

(ii) If $t \leq-l$, then $\bar{H}^{i}=0$ for $i=0, \ldots, \min (2, h-1)$ and

$$
\bar{H}^{i}= \begin{cases}S_{\frac{i-1}{2}-t-l}\left(H^{*}\right) \otimes S_{\frac{i-1}{2}}(C) & \text { if } 3 \leq i<h, i \neq \equiv 0(2), \\ & \text { if } 3 \leq i<h, i \equiv 0(2) .\end{cases}
$$

REMARK 5.7. In view of what we will do in the next section, we record here another interpretation of the complex $\mathcal{N}(t)$.

With the notion introduced above set $M=$ Coker $\psi^{*}$. By $\bar{\lambda}: M \rightarrow H^{*}$ we denote the linear map induced by $\varphi^{*}$. Then there is a canonical complex isomorphism

$$
\mathcal{N}(t) \longrightarrow\left(\mathcal{C}_{\bar{\lambda}}(t)\right)^{*}
$$


To see this we substitute $\varphi^{*}$ for $\psi$ in Proposition 3.5. We then obtain a natural complex isomorphism

$$
\mathcal{D}_{\varphi}(t) \stackrel{\tau}{\longrightarrow}\left(\mathcal{C}_{\varphi^{*}}(t)\right)^{*}
$$

Obviously $\left(\mathcal{C}_{\bar{\lambda}}(t)\right)^{*}$ may be viewed as a subcomplex of $\left(\mathcal{C}_{\varphi^{*}}(t)\right)^{*}$, and

$$
\tau(\mathcal{N}(t))=\left(\mathcal{C}_{\bar{\lambda}}(t)\right)^{*}
$$

since

$$
\operatorname{Ker}\left(\left(\bigwedge^{p} G^{*}\right)^{*} \stackrel{\left(d_{\psi^{*}}\right)^{*}}{\rightarrow}\left(F^{*} \otimes \bigwedge^{p-1} G^{*}\right)^{*}\right) \cong\left(\bigwedge^{p} M\right)^{*}
$$

\section{Koszul complexes in projective dimension one}

We shall now investigate the homology of the generalized Koszul complexes in free (projective) dimension 1 .

In this section $R$ is a noetherian ring, and $M$ an $R$-module which has a presentation

$$
0 \longrightarrow \mathcal{F} \stackrel{\chi}{\longrightarrow} \mathcal{G} \longrightarrow M \longrightarrow 0
$$

where $\mathcal{F}, \mathcal{G}$ are free modules of ranks $m$ and $n$. Then in particular $r=n-m \geq 0$.

In the sequel we consider $R$-homomorphisms $\bar{\lambda}: M \rightarrow \mathcal{H}$ into a finite free $R$ module $\mathcal{H}$ of rank $l \leq n$. By $\lambda$ we denote the corresponding lifted maps $\mathcal{G} \rightarrow \mathcal{H}$. Dualizing $\mathcal{F} \stackrel{\chi}{\rightarrow} \mathcal{G} \stackrel{\lambda}{\rightarrow} \mathcal{H}$ we go back to the situation previously studied. So we set $F=\mathcal{F}^{*}, G=\mathcal{G}^{*}, H=\mathcal{H}^{*}, \psi=\chi^{*}, \varphi=\lambda^{*}$, and $C=$ Coker $\psi$.

We say that a homomorphism of finite free $R$-modules is minimal if the entries of a representing matrix generate a proper ideal of $R$.

THEOREM 6.1. Set $\rho=r-l$ and $k=r+1-g$. Suppose that $g=$ grade $I_{\chi}>|\rho|+1$.

(a) $I_{\lambda} \subset \operatorname{Rad} I_{\chi}$, and in particular grade $I_{\lambda} \leq g$.

(b) The following conditions are equivalent:

(1) $\operatorname{grade} I_{\lambda}>|\rho|+1$;

(2) $\operatorname{Rad} I_{\chi}=\operatorname{Rad} I_{\lambda}$.

(c) Suppose that $I_{\chi} \neq R$ and that there is a $\bar{\lambda}$ such that grade $I_{\lambda}>|\rho|+1$. Then $l=k+1, r \geq l$, and $r-k$ is odd.

$\left(\mathrm{c}_{1}\right)$ Let $r=k+1(=l)$. The sequence $0 \rightarrow \mathcal{F} \stackrel{\chi}{\rightarrow} \mathcal{G} \stackrel{\lambda}{\rightarrow} \mathcal{H}$ as well as its dual $0 \rightarrow H \stackrel{\varphi}{\rightarrow} G \stackrel{\psi}{\rightarrow} F$ are exact. This can occur only if $I_{\chi}=I_{\lambda}$. Furthermore $m=1$ occurs if and only if the ith entry of a matrix for $\chi$ is $(-1)^{i}$ times the minor of a matrix for $\lambda$ by cancelling the ith row. 
$\left(\mathrm{c}_{2}\right)$ Let $r \geq k+3$ (i. e. $r \geq l+2$ ). If $\chi$ is minimal, then $m \leq k+1(=l)$. If $\lambda$ is minimal, then $m>k(=l-1)$.

Proof. (a) With respect to the assumption, Proposition [5.1.(2) yields $I_{\lambda}=I_{\lambda^{*}} \subset$ $\operatorname{Rad} I_{\chi^{*}}=\operatorname{Rad} I_{\chi}$.

(b), (1) $\Rightarrow(2)$ is an immediate consequence of Proposition 5.1.(2) while (2) $\Rightarrow(1)$ is trivial.

Next we prove the main statement of (c). From (b) we draw that $\operatorname{Rad} I_{\chi}=$ $\operatorname{Rad} I_{\lambda}$. Since $h:=$ grade $I_{\lambda}=g>1$, Proposition [5.1.(1) implies that $\rho \geq 0$ so $r \geq l$, and $r-k+1=g>\rho+1=r-l+1 \geq 1$ implies that $l>k$ and $r-k>0$.

Assume that $r-k$ is even. Then $r-k \geq 2$. Consider the complex $\mathcal{N}\left(\frac{r-k}{2}\right)$ defined in the previous section, and observe that

$$
N^{r-k+1}\left(\frac{r-k}{2}\right)=S_{\frac{r-k}{2}}\left(H^{*}\right) \otimes\left(\bigwedge^{r+l-k} M\right)^{*}
$$

since

$$
N^{r-k+1}\left(\frac{r-k}{2}\right)=\operatorname{Ker}\left(S_{\frac{r-k}{2}}\left(H^{*}\right) \otimes \bigwedge^{r+l-k} G \rightarrow S_{\frac{r-k}{2}}\left(H^{*}\right) \otimes \bigwedge^{r+l-k-1} G \otimes F\right) .
$$

Now $l>k$ implies that $\left(\bigwedge^{r+l-k} M\right)^{*}=0$. So $N^{r-k+1}\left(\frac{r-k}{2}\right)=0$. It follows that $\bar{H}^{r-k+1}\left(\frac{r-k}{2}\right)=0$. On the other hand, using Theorem [5.3 (a) we obtain an exact sequence

$$
0 \longrightarrow D_{0}(H) \otimes S_{\frac{r-k}{2}}(C) \longrightarrow \bar{H}^{r-k+1}\left(\frac{r-k}{2}\right) .
$$

Because $\bar{H}^{r-k+1}\left(\frac{r-k}{2}\right)=0$, we get $D_{0}(H) \otimes S_{\frac{r-k}{2}}(C)=0$ and consequently $C=0$ which is in contradiction with $I_{\chi} \neq R$. So $r-k$ must be odd.

If $r-k=1$, then $l=k+1$ since $l \leq r$. Suppose that $r-k \geq 3$ (and odd). Then

$$
N^{r-k}\left(\frac{r-k-1}{2}\right)=S_{\frac{r-k-1}{2}}\left(H^{*}\right) \otimes\left(\bigwedge^{r+l-k-1} M\right)^{*} .
$$

On the other hand we draw from Theorem 5.3 (a) that

$$
\bar{H}^{r-k}\left(\frac{r-k-1}{2}\right)=D_{0}(H) \otimes S_{\frac{r-k-1}{2}}(C) .
$$

If $l>k+1$, then $\left(\bigwedge^{r+l-k-1} M\right)^{*}=0$, so $S_{\frac{r-k-1}{2}}(C)=0$, a contradiction. It follows that $l=k+1$.

$\left(c_{1}\right)$ The first statement is an immediate consequence of the Buchsbaum-Eisenbud acyclicity criterion (see [E], Theorem 20.9). The second statement is also due to Buchsbaum and Eisenbud (see [N], Ch. 7, Theorem 3, or Corollary 5.1 in [BE1]). (Of course the third statement is a special case of the Theorem of Hilbert-Burch.)

To prove the first claim of $\left(\mathrm{c}_{2}\right)$ let $\chi$ be minimal. Localize at a prime ideal $P$ which contains $I_{1}(\chi)$. Then $g \leq$ grade $I_{\chi} R_{P}$. But $g<$ grade $I_{\chi} R_{P}$ is impossible: otherwise, since $I_{\lambda} \subset \operatorname{Rad} I_{\chi} \subset P$ in view of (a), we have grade $I_{\lambda} R_{P}>|\rho|+1$ 
and consequently $l=r+1-$ grade $I_{\chi} R_{P}+1 \leq k$ in contradiction with the first claim under (c). So we may assume $R$ to be local. Since $r \geq k+3, l=k+1$ and $M$ is free in depth 1 , we get $N^{r-k}\left(\frac{r-k-1}{2}\right)=S_{\frac{r-k-1}{2}}\left(H^{*}\right)$ and $N^{r-k+1}\left(\frac{r-k-1}{2}\right)=0$. So $\bar{H}^{r-k}\left(\frac{r-k-1}{2}\right)=S_{\frac{r-k-1}{2}}(C)$ is a quotient of $S_{\frac{r-k-1}{2}}\left(H^{*}\right)$. Therefore the minimal number of generators of $C$ cannot be greater than the minimal number of generators of $H$. It follows that $m \leq l=k+1$.

Since $r \geq k+3$ and $l=k+1$, we deduce that $\rho \geq 2$. So $h=g \geq 4$. To prove the second statement of $\left(\mathrm{c}_{2}\right)$ we dualize the sequence $0 \rightarrow \mathcal{F} \stackrel{\chi}{\rightarrow} \mathcal{G} \stackrel{\lambda}{\rightarrow} \mathcal{H}$. Set $r^{\prime}=n-l$ and $k^{\prime}=r^{\prime}+1-h$. From the first claim under (c) we draw that $m=k^{\prime}+1$. Since $h \geq 4$, we get $r^{\prime} \geq k^{\prime}+3$. Now let $\lambda$ be minimal. Applying the first part of $\left(\mathrm{c}_{2}\right)$, we obtain $(k+1=) l \leq k^{\prime}+1=m$.

COROLLARY 6.2. Set $\rho=r-l$ and suppose that $\infty>\operatorname{grade} I_{\chi}>|\rho|+1$. Then grade $I_{\lambda} \leq|\rho|+2$ for every $\bar{\lambda}$. Moreover, if grade $I_{\lambda}=|\rho|+2$, then grade $I_{\chi}$, grade $I_{\lambda}$, and $\rho$ are even.

Proof. If $\infty>\operatorname{grade} I_{\lambda}>|\rho|+1$, then the above theorem implies that grade $I_{\lambda}=$ grade $I_{\chi}=r+1-k=r-l+2=|\rho|+2$. Since $r-k$ is odd, the integers grade $I_{\chi}$, grade $I_{\lambda}, \rho$ must be even.

REMARK 6.3. Let $k \in \mathbb{N}$. If $l=k+1$ and $r-k>0$ is odd, then, in the cases listed under (c), there are always maps $\chi: R^{m} \rightarrow R^{n}$ and $\lambda: R^{n} \rightarrow R^{l}$ such that $\lambda \chi=0$ and grade $I_{\chi}=$ grade $I_{\lambda}=r-k+1$, provided there is a regular sequence of length $r-k+1$ in $R$. (See $[\mathrm{I}]$, section 3.2 for an example.)

The following criterion could occasionally be useful.

COROLLARY 6.4. Let $l \leq n, m \leq n$ and set $\rho=n-m-l$. Furthermore let $\mathcal{A}$ be an $l \times n$-matrix and $\mathcal{B}$ be an $n \times$ m-matrix with entries in $R$. Set $h=\operatorname{grade} I_{\mathcal{A}}$, $g=\operatorname{grade} I_{\mathcal{B}}$, and suppose that $\infty>h, g>|\rho|+1$. Then $\mathcal{A B} \neq 0$ in each of the following cases:

(1) $h \neq g$;

(2) $\rho$ odd;

(3) $g \neq|\rho|+2$;

(4) the ideals generated by the entries of $\mathcal{A}$ and $\mathcal{B}$ are proper ideals of $R$ and $l \neq m$ and $l \neq n-m$.

In the second part of this section we treat the case in which grade $I_{\chi}$ has the greatest possible value $n-m+1$.

The following result may be seen as an extension of the Hilbert-Burch Theorem.

THEOREM 6.5. Suppose that grade $I_{\chi}=r+1$. Then $I_{\lambda} \subset I_{\chi}$, and in particular grade $I_{\lambda} \leq r+1$. Set $\rho=r-l$. 
(a) If there is a $\bar{\lambda}$ such that grade $I_{\lambda}>|\rho|+1$, then $l=1$ and $r$ is odd.

(b) Suppose in addition that $\chi$ is minimal. Then the following are equivalent:

(1) There is a $\bar{\lambda}$ such that grade $I_{\lambda}>|\rho|+1$;

(2) $l=1$ and (i) $r=1$ or (ii) $m=1$ and $r \geq 3$ is odd.

(c) The following conditions are equivalent:

(1') grade $I_{\lambda}>|\rho|+1$;

(2') $I_{\lambda}=I_{\chi}$.

Proof. By Proposition [5.1.(3) we obtain $I_{\lambda}=I_{\lambda^{*}} \subset I_{\chi^{*}}=I_{\chi}$. Since $I_{\chi} \neq R$ by assumption, the first part of the theorem is clear.

Theorem 6.1. (c) implies (a) and also the implication (1) $\Rightarrow(2)$ of (b) since $k=0$. The other direction of (b) is a simple exercise.

It remains to prove (c), (1') $\Rightarrow\left(2^{\prime}\right)$ (since $\left(2^{\prime}\right) \Rightarrow\left(1^{\prime}\right)$ is trivial): We may assume $R$ to be local, and, using the uniqueness of minimal free resolutions, we can easily reduce to the case in which $\chi$ is minimal. According to what we have proved already, it follows that $l=1$ and (i) $r=1$ or (ii) $m=1$ and $r \geq 3$ is odd. If $r=1$, then we can apply the Hilbert-Burch Theorem to get the desired equality $I_{\lambda}=I_{\chi}$. If $m=1$ we look at the exact sequence

$$
R \stackrel{\lambda^{*}}{\longrightarrow} \mathcal{G}^{*} \stackrel{\chi^{*}}{\longrightarrow} R .
$$

which satisfies the hypothesis of Proposition 5.1.(3) since grade $I_{\lambda}>n-m$ by assumption. So

$$
I_{\chi}=I_{\chi^{*}} \subset I_{\lambda^{*}}=I_{\lambda} .
$$

COROLLARY 6.6. Suppose that grade $I_{\chi}=r+1$. Then the following conditions are equivalent:

(1) there is a $\bar{\lambda}$ with grade $I_{\lambda}=n-l+1$;

(2) $l=1, m=1$ and $r \geq 1$ is odd.

Proof. Only $(1) \Rightarrow(2)$ requires a proof. First $h, g \geq 1$, so $\rho \geq 0$. Then we have $n-l+1>\rho+1$. Consequently $I_{\lambda}=I_{\chi}$. From Theorem 6.5. (a) we obtain that $l=1$ and $r \geq 1$ is odd. Since $r=n-l$, it follows that $m=1$.

COROLLARY 6.7. Suppose $R$ to be local and grade $I_{\chi}=r+1$. Then the following conditions are equivalent:

(1) there is a $\bar{\lambda}$ with grade $I_{\lambda}>|\rho|+1$; 
(2) $l=1$ and (i) $r=1$ or (ii) $M$ has a minimal resolution

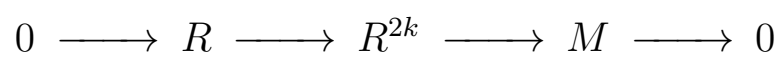

where $k \geq 2$.

Our next goal is the description of the homology of

$$
\begin{gathered}
\mathcal{C}_{\bar{\lambda}}(t): \cdots \rightarrow \bigwedge^{t+l+p} M \otimes S_{p}(\mathcal{H})^{*} \stackrel{\partial_{\bar{\lambda}}}{\rightarrow} \cdots \stackrel{\partial_{\bar{\lambda}}}{\rightarrow} \bigwedge^{t+l} M \otimes S_{0}(\mathcal{H})^{*} \stackrel{\nu_{\bar{\lambda}}}{\rightarrow} \bigwedge^{t} M \otimes S_{0}(\mathcal{H}) \stackrel{\partial_{\bar{\lambda}}}{\rightarrow} \\
\cdots \stackrel{\partial_{\bar{\lambda}}}{\rightarrow} \bigwedge^{0} M \otimes S_{t}(\mathcal{H}) \rightarrow 0 .
\end{gathered}
$$

For this purpose we refer to the the upper part of the Koszul bicomplex $\mathcal{K}_{., .}(t)$ of section 4 (the rows above the second row) which we denote by $\mathcal{B}_{., .}(t)$. We rewrite this complex as

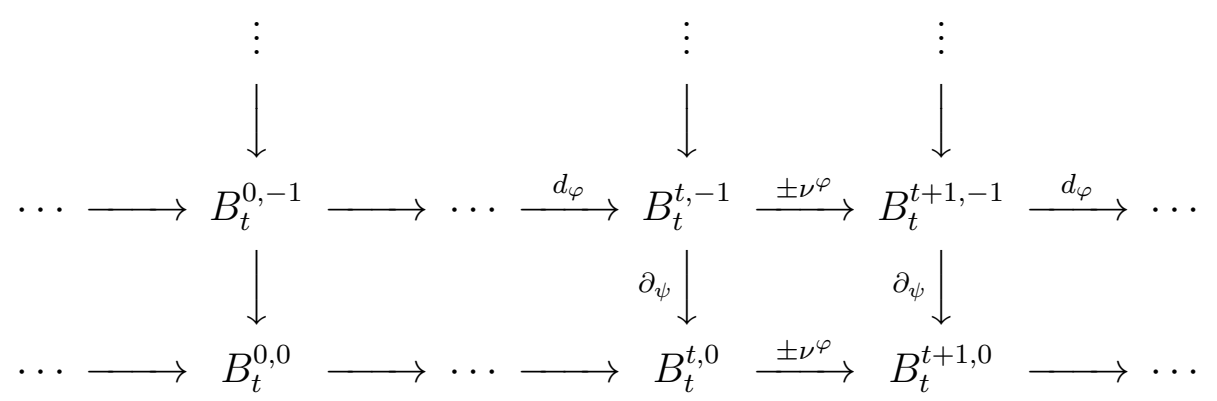

where

$$
B_{t}^{0,0}= \begin{cases}D_{t}(H) \otimes \bigwedge^{m} G \otimes S_{0}(F)^{*} & \text { if } 0 \leq t, \\ S_{0}\left(H^{*}\right) \otimes \bigwedge^{t+l+m} G \otimes S_{0}(F)^{*} & \text { if }-l \leq t<0, \\ S_{-t-l}\left(H^{*}\right) \otimes \bigwedge^{m} G \otimes S_{0}(F)^{*} & \text { if } t<-l .\end{cases}
$$

Set $M^{p}=\operatorname{Coker}\left(B_{t}^{p,-1} \stackrel{\partial_{\psi}}{\rightarrow} B_{t}^{p, 0}\right)$. The canonical surjection $B_{t}^{p, 0} \rightarrow M^{p}$ yields a complex morphism

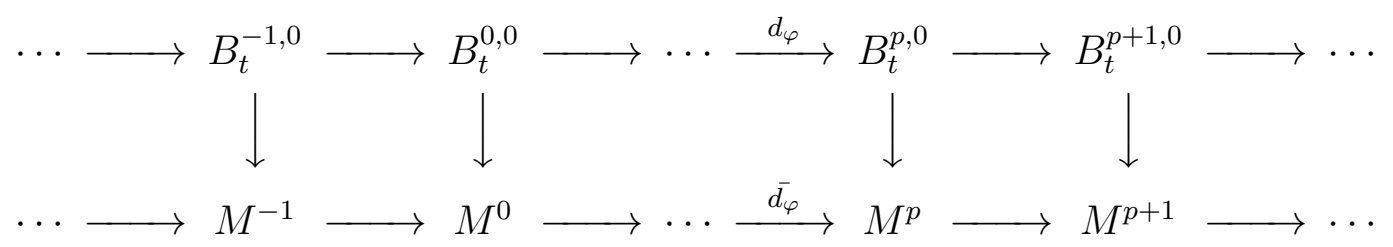

where the maps $\bar{d}_{\varphi}$ are induced by $d_{\varphi}$. The lower row is denoted by $\mathcal{M}(t)$.

We obtain an analogue with Remark 5.7.

PROPOSITION 6.8. Set $\rho=n-m-l$ as before. Then there is a (non-canonical) complex isomorphism

$$
\mathcal{M}(t) \longrightarrow \mathcal{C}_{\bar{\lambda}}(\rho-t)
$$


Proof. Corollary 3.3 combined with Proposition 3.5 provides (non-canonical) complex isomorphisms

(1) $\mathcal{D}_{\varphi}(t) \cong \mathcal{C}_{\varphi^{*}}(s-t)$ and

(2) $\mathcal{C}_{\psi}(t) \cong \mathcal{D}_{\psi^{*}}(r-t)$

where as above $s=n-l, r=n-m$. Next we consider the diagram

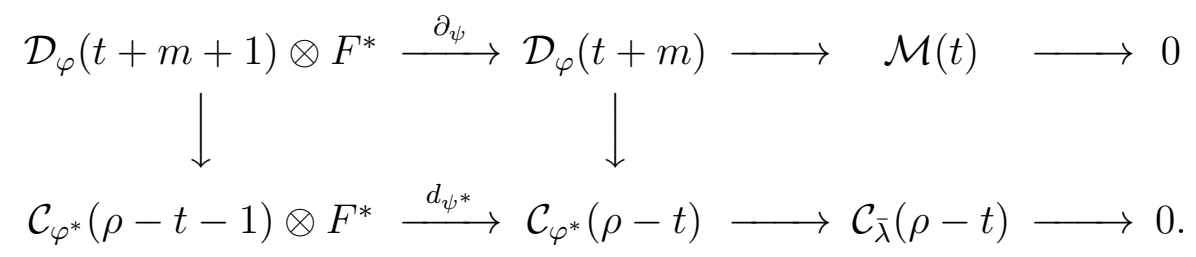

The isomorphisms (1) assure that the vertical arrows are isomorphisms, while (2) provides the commutativity of the diagram. The desired isomorphism is induced.

From the Koszul bicomplex in the previous section we extract the sequence of complexes

$$
\mathcal{D}_{\varphi}(t+m+1) \otimes F^{*} \stackrel{\partial_{\psi}^{-1}}{\longrightarrow} \mathcal{D}_{\varphi}(t+m) \stackrel{ \pm \nu_{\psi}}{\longrightarrow} \mathcal{D}_{\varphi}(t) \stackrel{\partial_{\psi}^{0}}{\longrightarrow} \mathcal{D}_{\varphi}(t-1) \otimes F
$$

Since $\mathcal{M}(t)=\operatorname{Coker}\left(B^{t,-1} \rightarrow B^{t, 0}\right)$ and $\mathcal{N}(t)=\operatorname{Ker}\left(C^{t, 0} \rightarrow C^{t, 1}\right)$, we obtain an induced complex morphism

$$
\mathcal{M}(t) \stackrel{\nu}{\longrightarrow} \mathcal{N}(t)
$$

This, composed with the inverse of the isomorphism of Proposition 6.8, yields a morphism

$$
\mathcal{C}_{\bar{\lambda}}(\rho-t) \stackrel{\mu}{\longrightarrow} \mathcal{N}(t) .
$$

Using Theorem 5.2 we easily deduce the following properties of $\mu$.

PROPOSITION 6.9. Let $r, l, g$ be as above.

(1) Suppose that $t+l \leq 0$ or $r<t+l$. Then the $\mu_{i}$ are isomorphisms for $i>r+1-g$, and $\mu_{r+1-g}$ is injective.

(2) Suppose that $l \leq t+l \leq r$.

(i) If $r+1-g \leq t$, then the $\mu_{i}$ are isomorphisms for $i>r+1-g$, and $\mu_{r+1-g}$ is injective.

(ii) If $t+l \leq r+1-g$, then the $\mu_{i}$ are isomorphisms for $i>r+2-g-l$, and $\mu_{r+2-g-l}$ is injective.

(iii) If $t<r+1-g<t+l$, then the $\mu_{i}$ are isomorphisms for $i>t$.

(3) Suppose that $0<t+l<l$. Then the $\mu_{i}$ are isomorphisms for $i>\min (0, r+$ $1-g-l-t)$ and, if $r+1-g-l-t \geq 0$, then $\mu_{r+1-g-l-t}$ is injective. 
THEOREM 6.10. Suppose that $g \geq r+1$. With notation as above set $S_{0}(C)=R / I_{\chi}$. Equip $\mathcal{C}_{\bar{\lambda}}(t)$ with the graduation induced by the complex isomorphism $\mathcal{M}(\rho-t) \rightarrow$ $\mathcal{C}_{\bar{\lambda}}(t)$ of Proposition 6.8. Then for the homology $\widetilde{H}$ of $\mathcal{C}_{\bar{\lambda}}(t)$ the following holds:

(a) in case $t \leq \frac{\rho}{2}$,

$$
\widetilde{H}^{i}=\left\{\begin{array}{cl}
D_{\rho-t-\frac{i-1}{2}}\left(\mathcal{H}^{*}\right) \otimes S_{\frac{i-1}{2}}(C) & \text { if } 0 \leq i<h, i \neq 0(2), \\
0 & \text { if } 0 \leq i<h, i \equiv 0(2)
\end{array}\right.
$$

(b) in case $\frac{\rho}{2}<t \leq \rho$,

$$
\widetilde{H}^{i}= \begin{cases}D_{\rho-t-\frac{i-1}{2}}\left(\mathcal{H}^{*}\right) \otimes S_{\frac{i-1}{2}}(C) & \text { if } 0 \leq i<\min (h, 2(\rho-t+1)), i \neq 0(2), \\ S_{\frac{i-l}{2}-\rho+t-1}(\mathcal{H}) \otimes S_{\frac{i+l}{2}-1}(C) & \text { if } 2(\rho-t+1)+l \leq i<h, i-l \equiv 0(2), \\ 0 & \text { otherwise if } 0 \leq i<h ;\end{cases}
$$

(c) in case $\rho<t<r$,

$$
\widetilde{H}^{i}=\left\{\begin{array}{cl}
S_{\frac{i-r+t-1}{2}(\mathcal{H}) \otimes S_{\frac{i+r-t-1}{2}}(C)} & \text { if } r-t+1 \leq i<h, i+r-t \not \equiv 0(2), \\
0 & \text { otherwise if } 0 \leq i<h
\end{array}\right.
$$

(d) in case $r \leq t$,

$$
\widetilde{H}^{i}=\left\{\begin{array}{cl}
S_{\frac{i-1}{2}+t-r}(\mathcal{H}) \otimes S_{\frac{i-1}{2}}(C) & \text { if } 0 \leq i<h, i \neq \equiv(2), \\
0 & \text { if } 0 \leq i<h, i \equiv 0(2) .
\end{array}\right.
$$

Proof. If $h=0$, then there is nothing to prove. If $h \geq 1$ then $\rho \geq 0$ and $r \geq 1$.

We use the complex morphism $\mu: \mathcal{C}_{\bar{\lambda}}(t) \rightarrow \mathcal{N}$. $(\rho-t)$ from above which induces the following commutative diagram:

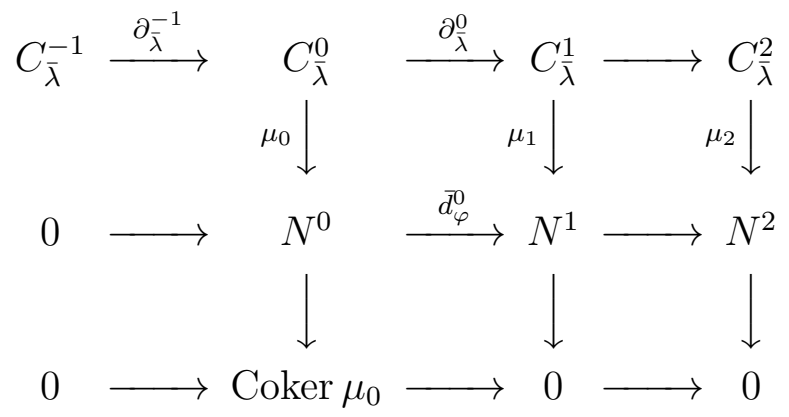

Since $C_{\bar{\lambda}}^{-1}$ is a torsion module and $\mu_{0}$ is injective, we have $\partial_{\bar{\lambda}}^{-1}=0$. If $h \geq 1$, then $d_{\varphi}^{0}$ is injective. This implies that $\partial_{\bar{\lambda}}^{0}$ is injective, so $\widetilde{H}^{0}=0$. If $h \geq 2$, Theorem 5.3 (or Theorem [5.6) says that the row homology at $N^{0}$ and at $N^{1}$ is 0 , so

$$
\widetilde{H}^{1}=\operatorname{Coker} \mu_{0}=\left\{\begin{array}{cl}
D_{\rho-t}\left(\mathcal{H}^{*}\right) \otimes R / I_{\chi} & \text { if } t \leq \rho, \\
0 & \text { if } \rho<i<r \\
S_{t-r}(\mathcal{H}) \otimes R / I_{\chi} & \text { if } r \leq t
\end{array}\right.
$$


If $h \geq 3$, then $\widetilde{H}^{2}$ equals the row homology at $N^{2}$. The remaining statements follow easily from Theorem 5.4 (or Theorem 5.6).

REMARK 6.11. The case $t=0$ in Theorem 6.10 covers Proposition 5.1 in [MNP].

COROLLARY 6.12. Suppose that $h=|\rho|+1$.

(1) If $l \geq \frac{r-1}{2}$, then $\left(\mathcal{C}_{\bar{\lambda}}(0)\right)^{*}$ has non-vanishing homology only in degree $\rho+1$, and if $r>1$, then

$$
\bar{H}^{0}(\rho+1)=S_{\rho}\left(\text { Coker } \chi^{*}\right)
$$

(2) If $l \geq \frac{r+1}{2}$, then the homology of $C_{\bar{\lambda}}(\rho+1)$ vanishes in positive degrees except for $\rho+1$, and

$$
\widetilde{H}^{\rho+1}(\rho+1)=S_{\rho+1}\left(\text { Coker } \chi^{*}\right)
$$

Proof. (a) Since $h \geq 1$, we have $\rho \geq 0$. In positive degrees, the homology of $\left(\mathcal{C}_{\bar{\lambda}}(0)\right)^{*}$ is almost the same as the homology of $\mathcal{C}_{\bar{\lambda}}(\rho)$, with the only exception in grade 1 (where the homology of $\left(\mathcal{C}_{\bar{\lambda}}(0)\right)^{*}$ is 0 ). If we require that $l \geq \frac{r-1}{2}$, Proposition 6.9. (2) provides the result.

(b) If $r=1$, then the claim is clear. If $r>1$, then $l>1$, and the result follows directly from Proposition 6.9. (3)

REMARK 6.13. Corollary 6.12,(1) was inspired by Lemma 5.5 in [MPN]. Corollary 6.12 (2) can be seen as an extension of Theorem [5.2.(b), since $l$ being big enough, one can easily deduce similar results.

REMARK 6.14. In the last part of this section we required for grade $I_{\chi}$ to have the greatest possible value. If we do the same for grade $I_{\lambda}$, we can obtain information about the homology of $\mathcal{C}_{\bar{\lambda}}(t)$, by studying the upper half of the Koszul bicomplex $\mathcal{K}_{., .}(t)$.

\section{References}

[BO1] N.Bourbaki. Algebra I, Chapters 1-3, Springer 1989.

[BO2] N.Bourbaki. Algèbre I, Chapitre 10, Algèbre homologique, Masson 1980.

[BE1] D. Buchsbaum and D. Eisenbud. Some Structure Theorems for Finite Free Resolutions. Advances in Math. 12 (1974), 84-139.

[BE2] D. Buchsbaum and D. Eisenbud. Generic Free Resolutions and a Family of Generically Perfect Ideals. Advances in Math. 18 (1975), 245-301.

[BE3] D. Buchsbaum and D. Eisenbud. Remarks on Ideals and Resolutions. Symposia Math. 11 (1973), 193-204. 
[BH] W.Bruns and J. Herzog. Cohen-Macaulay Rings. Cambridge Uni. Press 1996.

[BV1] W. Bruns and U. Vetter. Length formulas for the Local Cohomology of Exterior Powers. Math Z. 191 (1986), 145-158.

[BV2] W. Bruns and U. Vetter. Determinantal rings. Lect. Notes Math. 1327, Springer 1988.

[BV3] W. Bruns and U. Vetter. A Remark on Koszul Complexes. Beitr. Algebra Geom. 39 (1998), 249-254.

[BV4] W. Bruns and U. Vetter. The Koszul Complex in Projective Dimension One. Geometric and Combinatorial Aspects of Commutative Algebra, J. Herzog and G. Restuccia eds, Marcel Dekker 2001, 89-98.

[E] D. Eisenbud. Commutative Algebra with a View Toward Algebraic Geometry. Grad. Text in Math. 150, Springer 1995.

[HM] J. Herzog and A. Martsinkovsky. Glueing Cohen-Macaulay modules with applications to quasihomogeneous complete intersections with isolated singularities. Comment. Math. Helv. 68 (1993), 365-384.

[I] B. Ichim. Generalized Koszul Complexes. Thesis, Universität Oldenburg (Germany), 2004.

[MNP] J.C. Migliore, U. Nagel, and C. Peterson. Buchsbaum-Rim sheaves and their multiple sections. J. Algebra 219 (1999), 378-420.

[N] D.G. Northcott. Finite free resolutions. Cambridge Uni. Press 71, 1976. 